\title{
An Organocatalytic Two-atom Ring Expansion Approach to Optically Active Glutarimides
}

\author{
Yun-Long Wei, ${ }^{\text {a }}$ Yajun Ren, ${ }^{a}$ Damien Mailhol, ${ }^{a}$ Michel Rajzmann, \\ Jean Rodriguez, ${ }^{\mathrm{a}, *}$ and Yoann Coquerel ${ }^{\mathrm{a}, *}$ \\ a Aix Marseille Université, CNRS, Centrale Marseille, iSm2, 13397 Marseille, France \\ fax: + 33491289187 \\ E-mail: jean.rodriguez@univ-amu.fr; yoann.coquerel@univ-amu.fr
}

Abstract: An original two-step organocatalytic synthesis of optically active glutarimides from 2oxocyclobutane carboxamides is described featuring an isothiourea-catalyzed two-atom ring-expansive rearrangement.

Keywords: Nitrogen heterocycles; Organocatalysis; Rearrangement; Ring expansion; Strained molecules

The glutarimide moiety, that is to say the piperidine2,6-dione skeleton, can be found in many natural and non-natural products having various biological properties such as antibacterial, antitumoral and anti-inflammatory activities, and several marketed drugs contain a chiral glutarimide unit (Figure 1) ${ }^{[1]}$ While the synthesis of chiral glutarimides in the racemic series is now enabled at the industrial scale, there have been only few enantioselective methods reported to synthesize glutarimides in non-racemic forms. ${ }^{[2]}$ Actually, three approaches have been considered so far, relying either i) on the $\alpha$-functionalization of preformed glutarimides, ${ }^{[3]}$ ii) on $(3+3)$ annulation processes from secondary amides, ${ }^{[4]}$ and more originally iii) on the
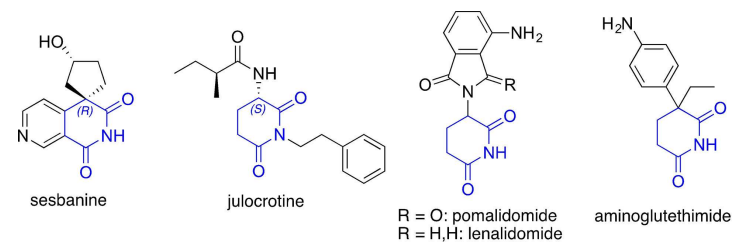

Figure 1. Selected structurally simple natural (left) and nonnatural (right) biologically active glutarimides. two-atom ring contraction of eight-membered benzazocinones (Scheme 1). ${ }^{[5]}$ Herein we propose a complementary organocatalytic approach that relies on a basecatalyzed ring rearrangement of 2-oxocyclobutane carboxamides resulting in a two-atom ring expansion (Scheme 1).

In early experiments, it was observed that the 2oxocyclobutane carboxamide 1 a could be catalytically rearranged into the corresponding glutarimide $\mathbf{2 a}$ ensuing a two-atom ring expansion, using the $\mathrm{N}$ heterocyclic carbene (NHC) IDipp as the catalyst (Scheme 2). Anecdotally, the four-membered ring substrate 1 a was conveniently prepared through the ring-contractive Wolff rearrangement of 2-diazo-cyclopentane-1,3-dione in the presence of 4-nitroaniline, ${ }^{[6]}$ and the synthesis of glutarimide $\mathbf{2} \mathbf{a}$ is thus based on a

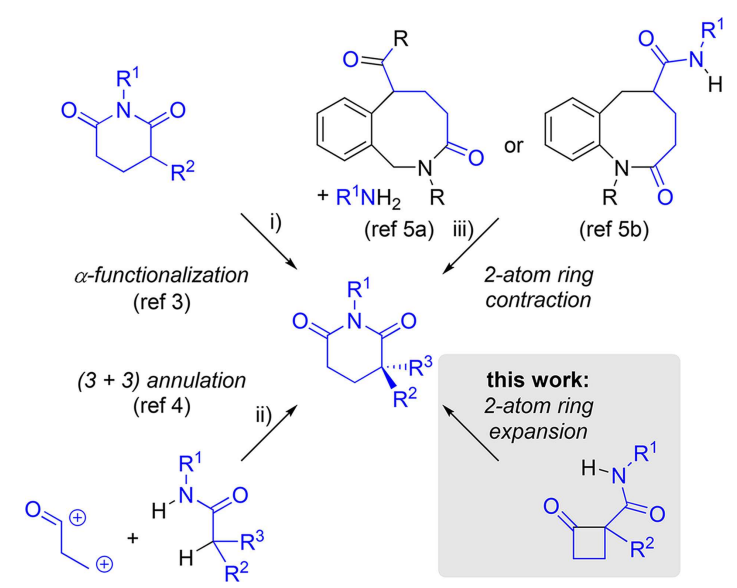

Scheme 1. Enantioselective synthetic approaches to glutarimides. 

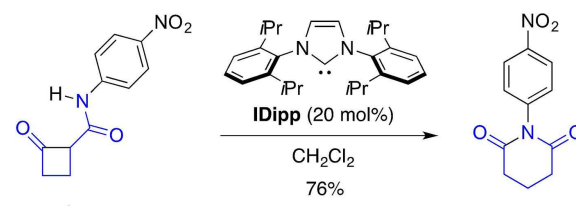

$76 \%$

2a

Scheme 2. Early observation of the base-catalyzed ring rearrangement of the 2-oxocyclobutane carboxamide $\mathbf{1}$ a into the glutarimide $\mathbf{2}$ a.

one-atom ring contraction/two-atom ring expansion sequence. From there, it was anticipated that a similar ring rearrangement would occur with the more complex and highly enantioenriched 2-oxocyclobutane carboxamide $\mathbf{3 a}$ derived from the enantioselective organocatalytic Michael addition of cyclobutanone 1 a to nitrostyrene catalyzed by the bifunctional aminocatalyst OC, as described in a previous article. ${ }^{[7]}$ Some representative basic catalysts were screened for the conversion of enantiopure $\mathbf{3} \mathbf{a}(\mathrm{er}>200: 1)$ into the corresponding glutarimide 4 a (Scheme 3 ). The $\mathrm{NHCs}$ IDipp and TPhPh afforded encouraging yields of the expected glutarimide product $\mathbf{4} \mathbf{a}$ as mixtures of the two possible diastereomers, albeit as nearly racemic materials, indicating a rapid racemization under these conditions. Similar observations resulted from the trials

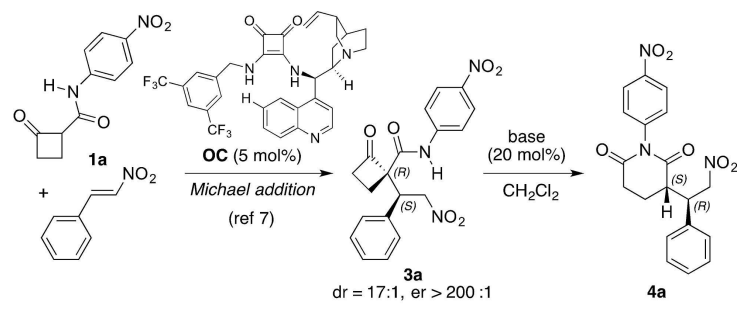

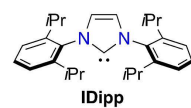

IDipp

$59 \%$ after $10 \mathrm{~h} @ 28{ }^{\circ} \mathrm{C}$
$\mathrm{dr}=16: 1, \mathrm{er}=1 \cdot 1$ and $16: 1$

$$
\stackrel{t B u-P}{t B}-\stackrel{t B u}{P}
$$

$\mathrm{dr}=1.7: 1$, er $=5.5: 1$ and $3.1: 1$

$$
\mathrm{Ph}
$$

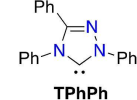

$60 \%$ after $24 \mathrm{~h} @ 20^{\circ} \mathrm{C}$
$\mathrm{dr}=2: 1$, er $=1.2: 1$ and $1.9: 1$

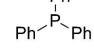

TPP

no reaction after
$24 \mathrm{~h} @ 20^{\circ} \mathrm{C}$
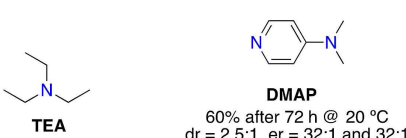

TEA $90 \%$ after $24 \mathrm{~h} @ 28{ }^{\circ} \mathrm{C}$
$\mathrm{dr}=1.1: 1$, er $=1.5: 1$ and $1.1: 1$

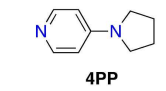

$\mathrm{PP}$

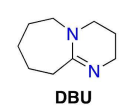

DBU

$90 \%$ after $24 \mathrm{~h} @ 28{ }^{\circ} \mathrm{C}$
$\mathrm{dr}=1: 1$, er $=21: 1$ and $16: 1$

degradation
after $4 \mathrm{~h} @ 28^{\circ} \mathrm{C}$
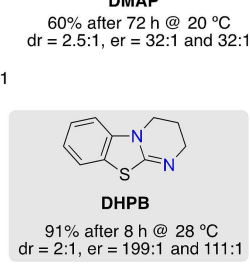

Scheme 3. Screening of basic catalysts for the ring rearrangement of 2-oxocyclobutane carboxamide 3 a. The major diastereomer of glutarimide $\mathbf{4 a}$ is depicted. with tri-tert-butylphosphine (TBP) and triethylamine (TEA). Interestingly, DMAP and its analog 4PP afforded both diastereomers of $\mathbf{4 a}$ with this time relatively good retentions of optical purity. In contrast, catalytic triphenylphosphine (TPP) or DBU did not allow the isolation of $\mathbf{4 a}$. Finally, it was found that $20 \mathrm{~mol} \%$ of $\mathbf{D H P B}^{[8]}$ is optimum to promote this ring rearrangement efficiently, allowing the isolation of $\mathbf{4 a}$ $(d r=2: 1)$ without significant loss of optical purity, indicating negligible racemization processes in that case. It was briefly explored if the two-step sequence could be performed in one-pot conditions, without isolating the intermediate Michael adduct $3 \mathbf{a}$. When $20 \mathrm{~mol} \%$ DHPB were added to the product reaction mixture of the first step, the glutarimide $\mathbf{4 a}(\mathrm{dr}=2.4: 1)$ was produced efficiently (quant. by ${ }^{1} \mathrm{H}$ NMR analysis) after 8 hours at $28^{\circ} \mathrm{C}$ but with important racemization (er=3.3:1 and 8.7:1), showing catalysts incompatibility and a preferred stepwise synthetic sequence.

The stereochemical outcome of the ring rearrangement reaction $\mathbf{3} \mathbf{a} \rightarrow \mathbf{4} \mathbf{a}$ was questioned. The issue of racemization was attributed to catalytic retro-Michael/ Michael processes of $\mathbf{3} \mathbf{a}$ involving the Brønsted base properties of the various achiral catalysts depicted in Scheme 3. This could be confirmed experimentally by a series of cross-experiments in the case of catalyst IDipp (Scheme 4).

Indeed, when the racemic glutarimide $4 \mathbf{a}$ was allowed to react with the nitroolefin having an extra 4methyl group on the benzene ring in the presence of IDipp or DHPB, no reaction occurred and 4 a was recovered quantitatively. This indicates that no retroMichael/Michael process is occurring on the glutarimide product $\mathbf{4}$ a under these conditions. In contrast, when the racemic 2-oxocyclobutane carboxamide $\mathbf{3 a}$

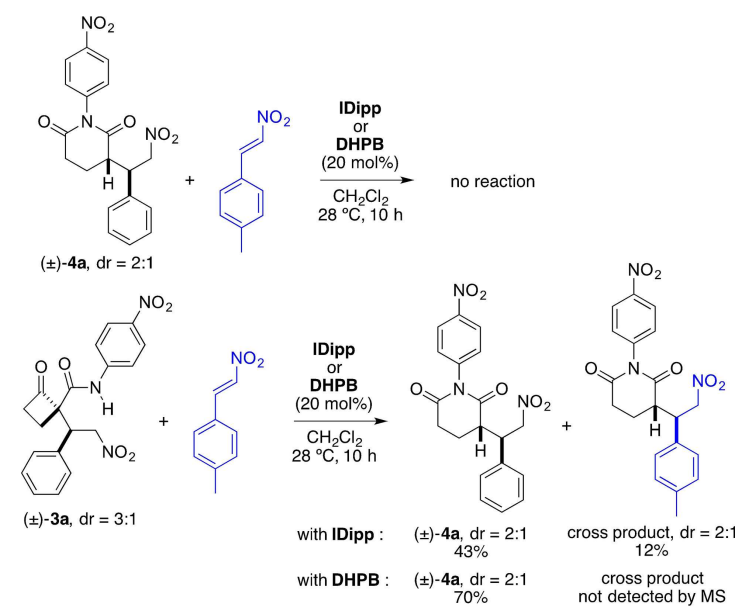

Scheme 4. Cross-experiments probing retro-Michael/Michael racemization processes of $\mathbf{3}$ a with IDipp and not with DHPB. The major diastereomers are depicted. 
was employed as the substrate with the IDipp catalyst under similar conditions, the glutarimide 4 a (43\%) could be obtained together with the cross-product glutarimide incorporating the nitroolefin having an extra 4-methyl group (12\%). These experiments demonstrate that IDipp-catalyzed retro-Michael/Michael processes occurred prior to the ring rearrangement of 3 a. ${ }^{[9]}$ Similar cross-experiments were performed with DHPB instead of IDipp and, as expected, no crossproduct could be detected in these cases showing that DHPB do not catalyze retro-Michael/Michael processes from $3 \mathbf{a}$ at a significant rate. The low diastereoselectivity of the transformation $\mathbf{3} \mathbf{a} \rightarrow \mathbf{4} \mathbf{a}$ is seemingly governed by thermodynamics due to relatively rapid DHPB-catalyzed epimerization at the imide enolizable position in product $\mathbf{4} \mathbf{a}$ as recently observed in closely related cases. ${ }^{[5 b, 10]}$ Supportive to this hypothesis, the relative Gibbs free energies of $(S, R)-\mathbf{4} \mathbf{a}$ and its diastereomer $(R, R)$-epi-4a were calculated using several density functional theory (DFT) methods, indicating that $\mathbf{4 a}$ is the thermodynamic diastereomer, though by only $2.2-3.6 \mathrm{~kJ}^{\mathrm{mol}}{ }^{-1}$ corresponding to a 2.4-4.3:1 ratio at equilibrium (see Supporting Information).

The scope and functional group tolerance of the glutarimides accessible by this approach were explored, first focusing on the electronics of the secondary amide group in cyclobutanones $\mathbf{3}$ (Scheme 5). It turned out that the two-atom ringexpansive rearrangement is only operative with electron-withdrawing $N$-aryl amide $\mathrm{R}^{1}$ substituents, affording the glutarimides $\mathbf{4 a - e}$ in good yields and high enantioselectivities. In contrast, cyclobutanones $3 \mathbf{f}-\mathbf{h}$ having electron-donating amide substituents were unreactive toward DHPB. Then, some representative nitroolefins were screened, which afforded the glutarimides $4 \mathbf{i}-\mathbf{0}$ having various aryl, heteroaryl or alkyl $\mathrm{R}^{2}$ substituents. The observed slight erosions of er in products $4 \mathbf{b}-\mathbf{e}$ and $4 \mathbf{i}-\mathbf{0}$ were attributed to DHPBcatalyzed retro-Michael/Michael processes from the 2oxocyclobutane carboxamides $\mathbf{3} \mathbf{b}-\mathbf{e}$ and $\mathbf{3} \mathbf{i}-\mathbf{o}$, respectively, as demonstrated above for $\mathbf{3}$ a with IDipp (see Scheme 4). Overall, an original enantioselective twostep approach to synthesize optically active glutarimides from 2-oxocyclobutane carboxamides has been discovered based on a two-step Michael/ring rearrangement sequence resulting in a two-atom ring expansion. Using this method, the glutarimide products described herein were generally obtained in good yields and high enantioselectivities, albeit generally as ca. 2:1 mixtures of the two possible diastereomers.

Mechanistically, two distinct scenarios are plausible to account for the observed ring rearrangement $\mathbf{3} \rightarrow \mathbf{4}$ : i) the basic catalyst is acting as a Brønsted base $\mathbf{B B}$ generating the corresponding amide isothiouronium salt of $\mathbf{3}$ to trigger an anionic ring closing/ring expansion sequence leading to 4 (Scheme 6, left), or ii)

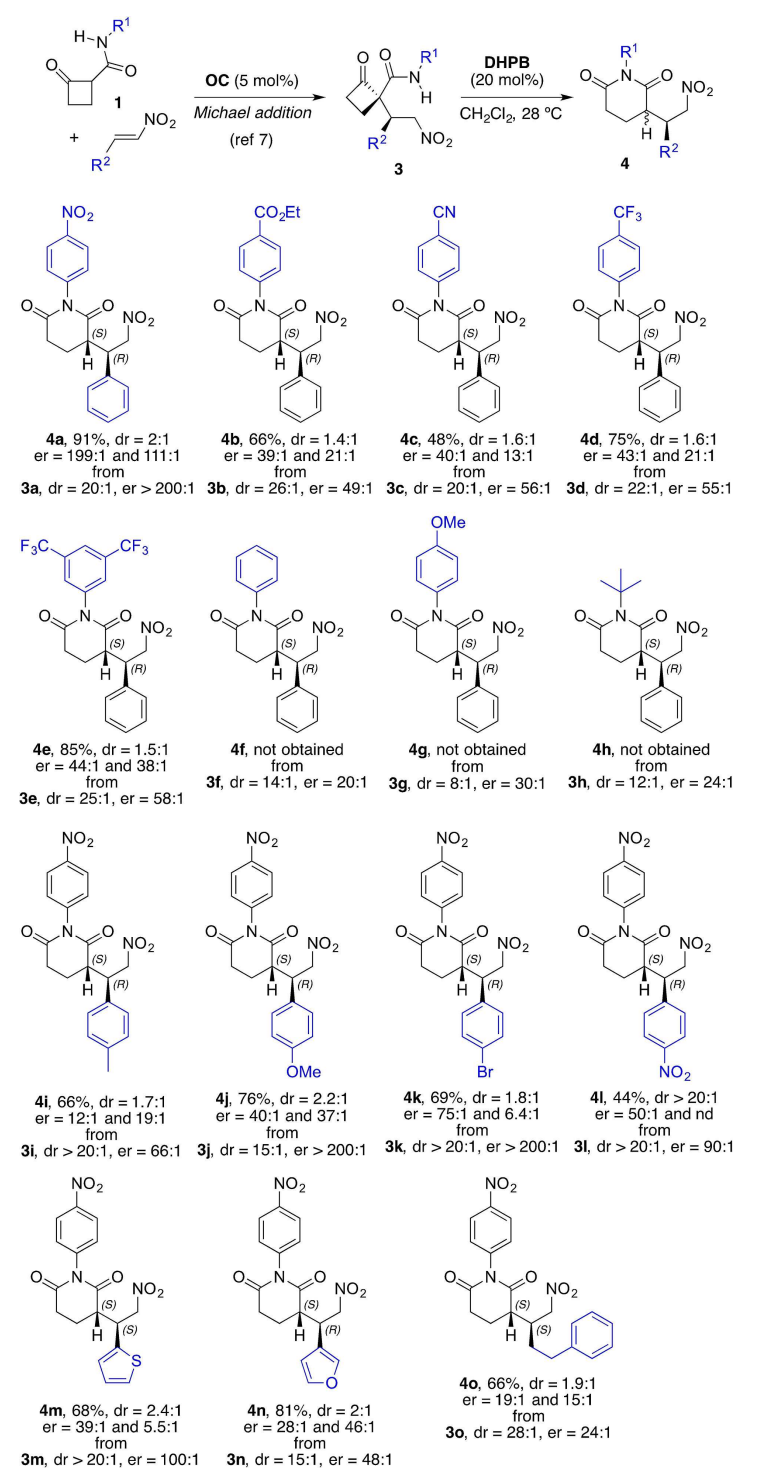

Scheme 5. Scope of the reaction. The major diastereomers are depicted. $\mathrm{nd}=$ not determined.

the basic catalyst is acting as a nucleophilic Lewis base LB promoting a ring opening/ring closing sequence to give 4 (Scheme 6, right). With the intention to gain insight in the intimate mechanism of this original transformation with DHPB as the catalyst, we investigated these two scenarios by computational modelization using DFT methods at an appropriate level of theory (see Supporting Information for details). The model transformations $\mathbf{I} \rightarrow \mathbf{V}\left(\mathrm{R}=\mathrm{NO}_{2}\right.$ and $\mathrm{OMe}$ ) were selected for this part of the study, and the mechanism involving DHPB as a Brønsted base was first considered (Scheme 7). As it could be anticipated, 


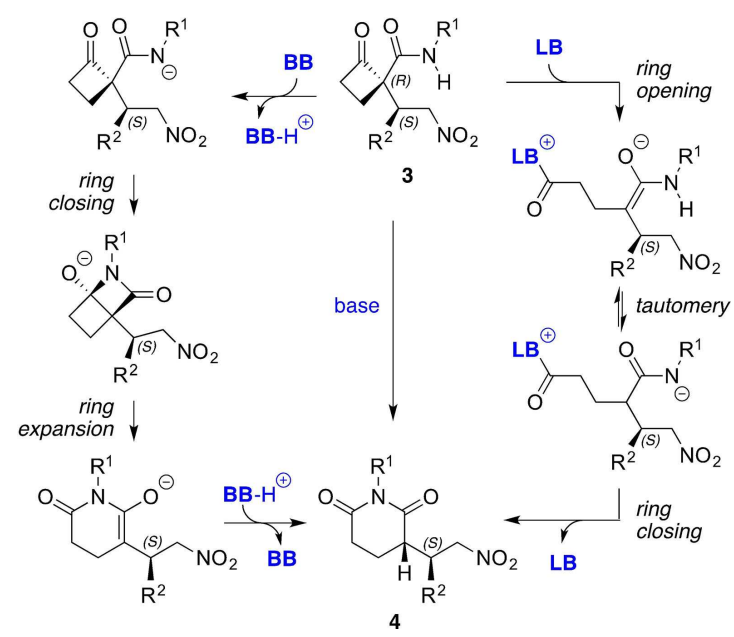

Scheme 6. Plausible mechanistic scenarios with the catalyst acting as a Brønsted base BB (left) or a nucleophilic Lewis base LB (right).

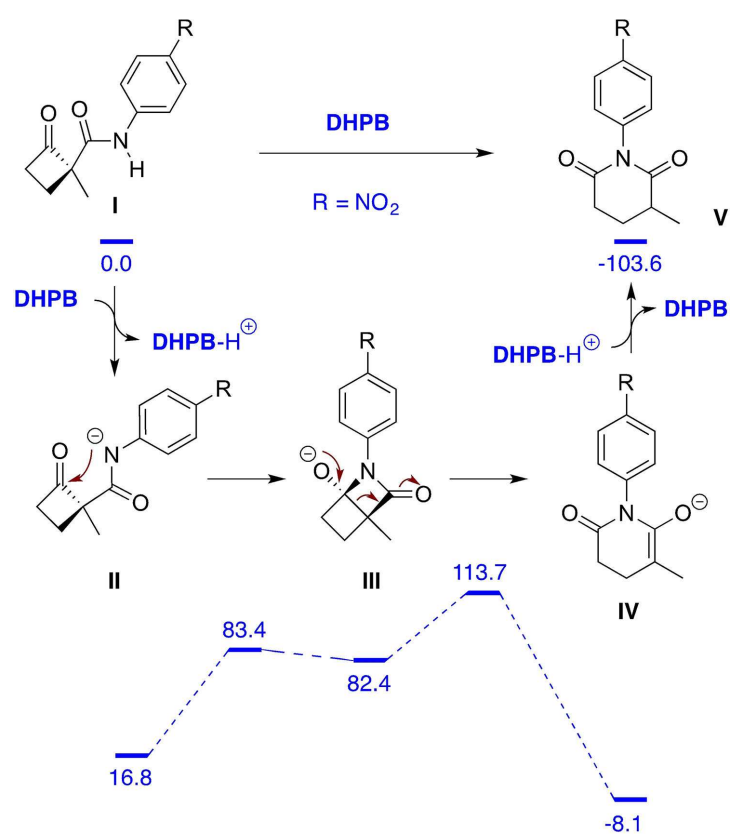

Scheme 7. Calculated energy profile for the model transformation $\mathbf{I} \rightarrow \mathbf{V}\left(\mathrm{R}=\mathrm{NO}_{2}\right)$ with $\mathbf{D H P B}$ employed as a catalytic Brønsted base [DFT, M06-2X-D/6-311+ + G(d,p) including a solvation model for dichloromethane]. Energies are Gibbs free energies expressed in $\mathrm{kJ} . \mathrm{mol}^{-1}$.

the ring rearrangement of the anion II directly derived from $\mathbf{I}$ into the anion $\mathbf{I V}$ precursor of $\mathbf{V}$ was found a two-step process proceeding via the bicyclic intermediate III. The reaction barrier of the overall rearrange-

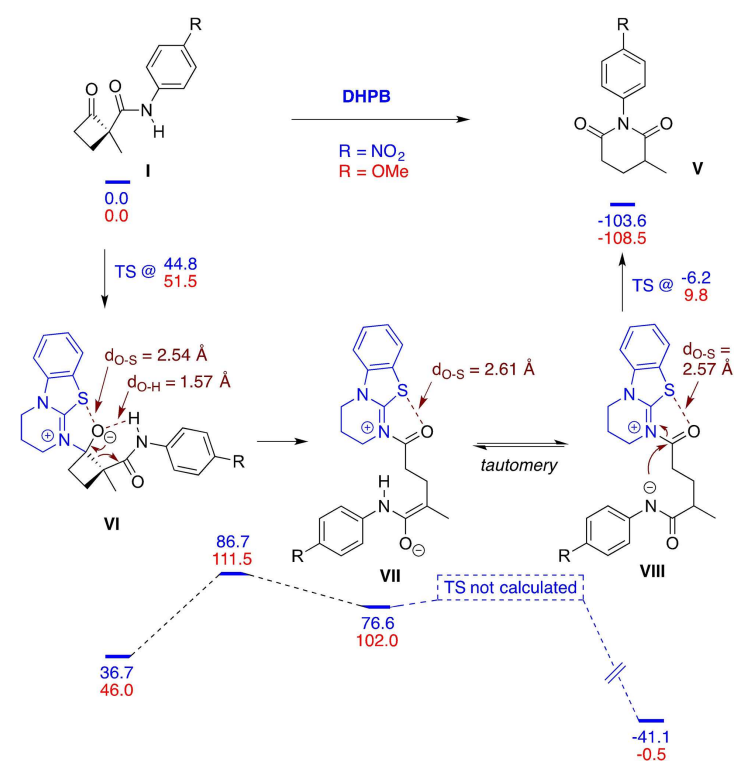

Scheme 8. Calculated energy profile for the model transformation $\mathbf{I} \rightarrow \mathbf{V}\left(\mathrm{R}=\mathrm{NO}_{2}\right.$ and $\left.\mathrm{R}=\mathrm{OMe}\right)$ with $\mathbf{D H P B}$ employed as a catalytic Lewis base [DFT, M06-2X-D/6-311 + + G(d,p) including a solvation model for dichloromethane]. Energies are Gibbs free energies expressed in $\mathrm{kJ} . \mathrm{mol}^{-1}$, and significant interactions are highlighted in brown color with inter atomic distances provided for the $\mathrm{R}=\mathrm{NO}_{2}$ series.

ment was calculated at $+113.7 \mathrm{~kJ} \cdot \mathrm{mol}^{-1}$ from the substrate I with $\mathrm{R}=\mathrm{NO}_{2}$ (see Supporting Information for details). The alternative mechanism involving DHPB as a catalytic nucleophilic Lewis base was then examined (Scheme 8). In this case it was found that the reaction is initiated by the formation of the ephemeral tetrahedral intermediate VI resulting from the addition of DHPB to the ketone carbonyl group with relatively low barriers. The fragmentation of VI into the acyl isothiouronium intermediate VII was computed as the rate limiting step in both the $\mathrm{R}=\mathrm{NO}_{2}$ and $\mathrm{R}=\mathrm{OMe}$ series, with reaction barriers at +86.7 and $+111.5 \mathrm{~kJ} . \mathrm{mol}^{-1}$, respectively. Then, a thermodynamically favored tautomery can afford intermediate VIII with this time the negative charge located on the nitrogen atom of the amide group. The relatively important stabilization energies in the zwitterionic intermediates VIII $\left(\mathrm{R}=\mathrm{NO}_{2}\right.$ and $\left.\mathrm{OMe}\right)$ was attributed, at least in part, to strong $\pi$ donor-acceptor stabilizing interactions between the negatively charged $N$-aryl amide planar moiety and the positively charged acyl isothiouronium planar moiety, with interplanar distances of ca. $3.2 \AA$ for $\mathrm{R}=\mathrm{NO}_{2}$ and $3.4 \AA$ for $\mathrm{R}=\mathrm{OMe}$ (Figure 2). Finally, intermediate VIII can cyclize in an irreversible manner to afford the glutarimide product $\mathbf{V}$ and regenerate the catalyst. Overall, these calculations with DHPB as the catalyst indicate i) that both 

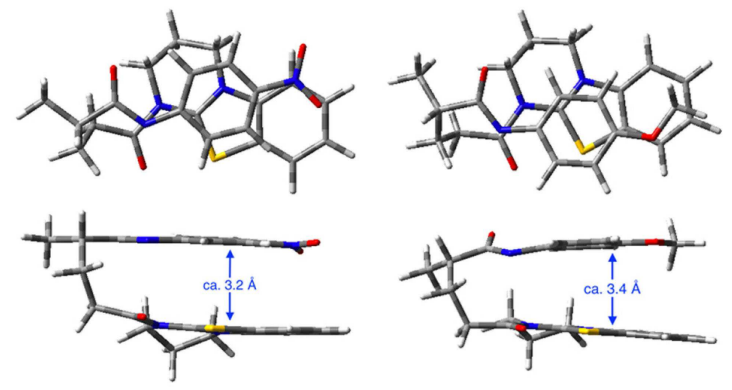

Figure 2. Top and side views of optimized geometries for intermediates VIII with $\mathrm{R}=\mathrm{NO} 2$ (left) and $\mathrm{R}=\mathrm{OMe}$ (right).

mechanisms are energetically possible, ii) that the mechanism involving the catalyst as a nucleophilic Lewis base is kinetically favored, and iii) that the reaction with $\mathrm{R}=\mathrm{OMe}$ would proceed at an extremely slow rate under the reaction conditions, which is in perfect agreement with the experimental results (see compound $\mathbf{4 g}$ not obtained in Scheme 5). In order to confirm this substituent effect, the analog of $\mathbf{1}$ a having a OMe substituent in place of the $\mathrm{NO}_{2}$ group was allowed to react with DHPB $(20 \mathrm{~mol} \%)$ in dichloromethane without promoting a detectable reaction after $14 \mathrm{~h}$. Overall, it seems that the ring rearrangement of 2-oxocyclobutane carboxamides into glutarimides catalyzed by DHPB described herein proceeded via acyl isothiouronium intermediates of type VII/VIII. ${ }^{[11]}$ Of course, these conclusions are only valid for DHPB, and it cannot be excluded that the other operative catalysts depicted in Scheme 3 behave otherwise. ${ }^{[12]}$

In summary, an original approach was discovered for the synthesis of optically active glutarimides. It is based on a two-step organocatalytic approach involving an enantioselective Michael addition of 2-oxocyclobutane carboxamides to nitroolefins using a chiral bifunctional aminocatalyst, followed by a two-atom ring-expansive rearrangement catalyzed by an achiral isothiourea catalyst. The glutarimide products prepared by this approach were generally obtained as mixtures of the two possible diastereomers in good yields and high enantioselectivities. Stereochemical outcomes were rationalized, and a computational mechanistic study showed that the ring rearrangement step is seemingly promoted by the nucleophilicity and Lewis basicity of the isothiourea catalyst.

\section{Experimental Section}

\section{General Information}

See the Supporting Information.

\section{Synthesis of 2-oxocyclobutane Carboxamides 3}

The syntheses and characterization data of optically active $\mathbf{3}$ a, $\mathbf{3 e}-\mathbf{h}$, and $\mathbf{3} \mathbf{j}-\mathbf{0}$ were reported in a previous article. ${ }^{[7]}$ Optically active $\mathbf{3} \mathbf{b}-\mathbf{d}$ and $\mathbf{3} \mathbf{i}$ were prepared following the same procedure and their characterization data are provided below. All racemic 2-oxocyclobutane carboxamides $3 \mathbf{a}-\mathbf{0}$ were prepared from the corresponding unsubstituted 2-oxocyclobutane carboxamides $\mathbf{1}^{[6 \mathrm{a}]}$ and the corresponding nitroolefins using the polystyrene-supported phosphazene catalyst known as PBEMP. ${ }^{[13]}$

Compound $3 \mathbf{b}$ : Following the general procedure, ${ }^{[7]}$ the reaction between the 2-oxocyclobutane carboxamide $1 \quad\left(\mathrm{R}^{1}=4\right.$ $\left.\mathrm{CO}_{2} \mathrm{Et}-\mathrm{C}_{6} \mathrm{H}_{4}, 100 \mathrm{mg}, 0.36 \mathrm{mmol}\right)$ and nitrostyrene (45 mg, $0.30 \mathrm{mmol})$ in anhydrous dichloromethane $(2 \mathrm{~mL})$ with catalyst OC (9 mg, $0.015 \mathrm{mmol})$ for $14 \mathrm{~h}$ afforded the 2-oxocyclobutane carboxamide $\mathbf{3 b}$ (94 $\mathrm{mg}, 76 \%$ yield, $\mathrm{dr}=26: 1$, er $=49: 1)$ as a white solid. $\boldsymbol{R}_{\boldsymbol{f}}=0.40$ (petrol ether/ethyl acetate, $2: 1$ ). $\mathbf{M p}=$ $182-184^{\circ} \mathrm{C}$ (amorphous). $[\boldsymbol{\alpha}]_{\mathbf{D}}{ }^{25}=-153.3 \quad\left(c=1.0, \mathrm{CHCl}_{3}\right)$. HRMS (ESI +$) \mathrm{m} / z$ calcd for $\mathrm{C}_{22} \mathrm{H}_{23} \mathrm{~N}_{2} \mathrm{O}_{6}{ }^{+}[\mathrm{M}+\mathrm{H}]^{+}=$ 411.1551, found $=411.1551$. ${ }^{1} \mathbf{H} \mathbf{N M R}\left(400 \mathrm{MHz}, \mathrm{CDCl}_{3}\right)$ major diastereomer: $\delta 8.26$ (br s, $1 \mathrm{H}), 8.02(\mathrm{~d}, J=8.4 \mathrm{~Hz}, 2 \mathrm{H})$, 7.57 (d, $J=8.8 \mathrm{~Hz}, 2 \mathrm{H}), 7.40-7.31(\mathrm{~m}, 3 \mathrm{H}), 7.30-7.26(\mathrm{~m}, 2 \mathrm{H})$, $4.97(\mathrm{dd}, J=14.0,10.6 \mathrm{~Hz}, 1 \mathrm{H}), 4.86$ (dd, $J=14.0,5.0 \mathrm{~Hz}$, $1 \mathrm{H}), 4.36$ (q, $J=7.1 \mathrm{~Hz}, 2 \mathrm{H}), 4.23(\mathrm{dd}, J=10.6,5.0 \mathrm{~Hz}, 1 \mathrm{H})$, 2.82 (ddd, $J=15.1,10.2,4.1 \mathrm{~Hz}, 1 \mathrm{H}), 2.63-2.45(\mathrm{~m}, 1 \mathrm{H}), 2.40-$ $2.17(\mathrm{~m}, 2 \mathrm{H}), 1.38(\mathrm{t}, \quad J=7.1 \mathrm{~Hz}, 3 \mathrm{H}) .{ }^{13} \mathbf{C}\left\{{ }^{1} \mathbf{H}\right\} \quad$ NMR $\left(100 \mathrm{MHz}, \mathrm{CDCl}_{3}\right)$ major diastereomer: $\delta 208.7(\mathrm{C}), 166.0(\mathrm{C})$, 165.7 (C), 140.9 (C), $133.4(\mathrm{C}), 130.9(2 \mathrm{CH}), 129.4(2 \mathrm{CH})$, $129.1(\mathrm{CH}), 128.5(2 \mathrm{CH}), 127.0(\mathrm{C}), 119.4(2 \mathrm{CH}), 76.2(\mathrm{C})$, $74.7\left(\mathrm{CH}_{2}\right), 61.1\left(\mathrm{CH}_{2}\right), 47.0(\mathrm{CH}), 44.5\left(\mathrm{CH}_{2}\right), 17.9\left(\mathrm{CH}_{2}\right)$, $14.4\left(\mathrm{CH}_{3}\right)$. HPLC: Chiralpak IE eluted with 1:1 heptane/ ethanol at $1 \mathrm{~mL} / \mathrm{min}$ at $25^{\circ} \mathrm{C}$, UV detection at $270 \mathrm{~nm}$, retention times: major enantiomer $7.88 \mathrm{~min}$, minor enantiomer $10.00 \mathrm{~min}$.

Compound $3 \mathrm{c}$ : Following the general procedure,${ }^{[7]}$ the reaction between the 2-oxocyclobutane carboxamide $1 \quad\left(\mathrm{R}^{1}=4\right.$ $\mathrm{CN}-\mathrm{C}_{6} \mathrm{H}_{4}, \quad 77 \mathrm{mg}, \quad 0.36 \mathrm{mmol}$ ) and nitrostyrene (45 mg, $0.30 \mathrm{mmol})$ in anhydrous dichloromethane $(2 \mathrm{~mL})$ with catalyst OC (9 mg, $0.015 \mathrm{mmol})$ for $14 \mathrm{~h}$ afforded the 2-oxocyclobutane carboxamide 3 c $(72 \mathrm{mg}, 66 \%$ yield, $\mathrm{dr}=20: 1$, er $=56: 1)$ as a white solid. $\boldsymbol{R}_{\boldsymbol{f}}=0.51$ (petrol ether/ethyl acetate, $2: 1$ ). $\mathbf{M p}=$ $136-138^{\circ} \mathrm{C}$ (amorphous). $[\boldsymbol{\alpha}]_{\mathrm{D}}{ }^{25}=-127.4 \quad\left(c=1.0, \mathrm{CHCl}_{3}\right)$. HRMS (ESI +$) \mathrm{m} / z$ calcd for $\mathrm{C}_{20} \mathrm{H}_{18} \mathrm{~N}_{3} \mathrm{O}_{4}{ }^{+}[\mathrm{M}+\mathrm{H}]^{+}=$ 364.1292, found $=364.1290$. ${ }^{1} \mathbf{H} \quad \mathbf{N M R}\left(400 \mathrm{MHz}, \mathrm{CDCl}_{3}\right)$ major diastereomer: $\delta 8.32$ (br s, $1 \mathrm{H}), 7.61$ (br s, 4H), 7.40$7.32(\mathrm{~m}, 3 \mathrm{H}), 7.29-7.26(\mathrm{~m}, 2 \mathrm{H}), 4.96(\mathrm{dd}, J=14.0,10.2 \mathrm{~Hz}$, $1 \mathrm{H}), 4.85$ (dd, $J=14.0,5.2 \mathrm{~Hz}, 1 \mathrm{H}), 4.24$ (dd, $J=10.2,5.2 \mathrm{~Hz}$, $1 \mathrm{H}), 2.82$ (ddd, $J=15.1,10.2,4.5 \mathrm{~Hz}, 1 \mathrm{H}), 2.63-2.44(\mathrm{~m}, 1 \mathrm{H})$, 2.43-2.18 (m, 2H). ${ }^{13} \mathbf{C}\left\{{ }^{1} \mathbf{H}\right\}$ NMR $\left(100 \mathrm{MHz}, \mathrm{CDCl}_{3}\right)$ major diastereomer: $\delta 208.5(\mathrm{C}), 165.9(\mathrm{C}), 140.8(\mathrm{C}), 133.3(2 \mathrm{CH})$, $133.3(\mathrm{C}), 129.4(2 \mathrm{CH}), 129.1(\mathrm{C}), 128.4(2 \mathrm{CH}), 120.1(2 \mathrm{CH})$, $118.6(\mathrm{CH}), 108.1(\mathrm{C}), 77.1(\mathrm{C}), 74.6\left(\mathrm{CH}_{2}\right), 46.8(\mathrm{CH}), 44.5$ $\left(\mathrm{CH}_{2}\right), 17.8\left(\mathrm{CH}_{2}\right)$. HPLC: Chiralpak IA eluted with $1: 1$ heptane/ethanol at $1 \mathrm{~mL} / \mathrm{min}$ at $25^{\circ} \mathrm{C}$, UV detection at $240 \mathrm{~nm}$, retention times: major enantiomer $6.07 \mathrm{~min}$, minor enantiomer $9.23 \mathrm{~min}$.

Compound $3 \mathrm{~d}$ : Following the general procedure, ${ }^{[7]}$ the reaction between the 2-oxocyclobutane carboxamide $1 \quad\left(\mathrm{R}^{1}=4\right.$ $\left.\mathrm{CF}_{3}-\mathrm{C}_{6} \mathrm{H}_{4}, 93 \mathrm{mg}, \quad 0.36 \mathrm{mmol}\right)$ and nitrostyrene $(45 \mathrm{mg}$, $0.30 \mathrm{mmol})$ in anhydrous dichloromethane $(2 \mathrm{~mL})$ with catalyst 
OC ( $9 \mathrm{mg}, 0.015 \mathrm{mmol})$ for $14 \mathrm{~h}$ afforded the 2-oxocyclobutane carboxamide $\mathbf{3 d}(100 \mathrm{mg}, 82 \%$ yield, $\mathrm{dr}=22: 1$, er $=55: 1)$ as a white solid. $\boldsymbol{R}_{\boldsymbol{f}}=0.60$ (petrol ether/ethyl acetate, 2:1). $\mathbf{M p}=$ $131-133^{\circ} \mathrm{C}$ (amorphous). $[\boldsymbol{\alpha}]_{\mathbf{D}}{ }^{25}=-124.6 \quad\left(c=1.0, \mathrm{CHCl}_{3}\right)$. HRMS $(\mathrm{ESI}+) \mathrm{m} / \mathrm{z}$ calcd for $\mathrm{C}_{20} \mathrm{H}_{18} \mathrm{~F}_{3} \mathrm{~N}_{2} \mathrm{O}_{4}{ }^{+}[\mathrm{M}+\mathrm{H}]^{+}=$ 407.1213, found $=407.1213$. ${ }^{1} \mathbf{H}$ NMR $\left(400 \mathrm{MHz}, \mathrm{CDCl}_{3}\right)$ major diastereomer: $\delta 8.25(\mathrm{br} \mathrm{s}, 1 \mathrm{H}), 7.56-7.64(\mathrm{~m}, 4 \mathrm{H}), 7.42$ $7.32(\mathrm{~m}, 3 \mathrm{H}), 7.28$ (dd, $J=7.7,1.7 \mathrm{~Hz}, 2 \mathrm{H}), 4.97$ (dd, $J=14.0$, $10.4 \mathrm{~Hz}, 1 \mathrm{H}), 4.87$ (dd, $J=14.0,5.1 \mathrm{~Hz}, 1 \mathrm{H}), 4.24$ (dd, $J=$ $10.3,5.1 \mathrm{~Hz}, 1 \mathrm{H}), 2.82$ (ddd, $J=15.1,10.2,4.1 \mathrm{~Hz}, 1 \mathrm{H}), 2.60$ $2.46(\mathrm{~m}, 1 \mathrm{H}), 2.41-2.19(\mathrm{~m}, 2 \mathrm{H}) .{ }^{13} \mathbf{C}\left\{{ }^{1} \mathbf{H}\right\}$ NMR $(100 \mathrm{MHz}$, $\mathrm{CDCl}_{3}$ ) major diastereomer: $\delta 208.7(\mathrm{C}), 165.9(\mathrm{C}), 139.9(\mathrm{C})$, $133.4(\mathrm{C}), 129.4(2 \mathrm{CH}), 129.1(\mathrm{CH}), 128.5(2 \mathrm{CH}), 126.9(\mathrm{q}$ $\left.J_{C-F}=28 \mathrm{~Hz}, \mathrm{C}\right), 126.4\left(\mathrm{q}, J_{C-F}=4 \mathrm{~Hz}, 2 \mathrm{CH}\right), 123.9\left(\mathrm{q}, J_{C-F}=\right.$ $270 \mathrm{~Hz}, \mathrm{C}), 120.0$ (2CH), $76.1(\mathrm{C}), 74.7\left(\mathrm{CH}_{2}\right), 47.0(\mathrm{CH}), 44.5$ $\left(\mathrm{CH}_{2}\right), 17.9\left(\mathrm{CH}_{2}\right),{ }^{19} \mathbf{F}\left\{{ }^{13} \mathbf{C}\right\}$ NMR $\left(282 \mathrm{MHz}, \mathrm{CDCl}_{3}\right): \delta-62.3$. HPLC: Chiralpak IA eluted with 7:3 heptane/ethanol at $1 \mathrm{~mL} /$ $\min$ at $25^{\circ} \mathrm{C}$, UV detection at $254 \mathrm{~nm}$, retention times: major enantiomer $6.33 \mathrm{~min}$, minor enantiomer $12.95 \mathrm{~min}$.

Compound $3 \mathrm{i}$ : Following the general procedure, ${ }^{[7]}$ the reaction between the 2-oxocyclobutane carboxamide $1 \quad\left(\mathrm{R}^{1}=4\right.$ $\mathrm{NO}_{2}-\mathrm{C}_{6} \mathrm{H}_{4}, 76 \mathrm{mg}, 0.33 \mathrm{mmol}$ ) and (E)-1-methyl-4-(2-nitrovinyl)benzene $(45 \mathrm{mg}, 0.27 \mathrm{mmol})$ in anhydrous dichloromethane $(2 \mathrm{~mL})$ with catalyst $\mathbf{O C}(8 \mathrm{mg}, 0.013 \mathrm{mmol})$ for $16 \mathrm{~h}$ afforded the 2-oxocyclobutane carboxamide $\mathbf{3 i}(70 \mathrm{mg}, 65 \%$ yield, $\mathrm{dr}>20: 1$, er $=66: 1$ ) as a white solid. $\boldsymbol{R}_{\boldsymbol{f}}=0.50$ (petrol ether/ethyl acetate, $2: 1)$. $\mathbf{M p}=81-83^{\circ} \mathrm{C}$ (amorphous). $[\boldsymbol{\alpha}]_{\mathbf{D}}{ }^{25}=$ $-85.5\left(c=0.5, \mathrm{CHCl}_{3}\right)$. HRMS $(\mathrm{ESI}+) \mathrm{m} / \mathrm{z}$ calcd for $\mathrm{C}_{20} \mathrm{H}_{20} \mathrm{~N}_{3} \mathrm{O}_{6}{ }^{+} \quad[\mathrm{M}+\mathrm{H}]^{+}=398.1347$, found $=398.1345 .{ }^{1} \mathbf{H}$ NMR $\left(400 \mathrm{MHz}, \mathrm{CDCl}_{3}\right.$ ) major diastereomer: $\delta 8.43$ (br s, $\left.1 \mathrm{H}\right)$, $8.24(\mathrm{~d}, J=9.0 \mathrm{~Hz}, 2 \mathrm{H}), 7.69$ (d, $J=9.0 \mathrm{~Hz}, 2 \mathrm{H}), 7.23-7.11(\mathrm{~m}$, $4 \mathrm{H}), 4.94(\mathrm{dd}, J=13.9,10.1 \mathrm{~Hz}, 1 \mathrm{H}), 4.84$ (dd, $J=13.9$, $5.3 \mathrm{~Hz}, 1 \mathrm{H}), 4.21$ (dd, $J=10.1,5.3 \mathrm{~Hz}, 1 \mathrm{H}), 2.84$ (ddd, $J=$ $18.2,10.1,4.3 \mathrm{~Hz}, 1 \mathrm{H}), 2.61-2.51(\mathrm{~m}, 1 \mathrm{H}), 2.43-2.23(\mathrm{~m}, 2 \mathrm{H})$ 2.33 (s, 3H). ${ }^{13} \mathbf{C}\left\{{ }^{1} \mathbf{H}\right\}$ NMR $\left(100 \mathrm{MHz}, \mathrm{CDCl}_{3}\right)$ major diastereomer: $\delta 208.9$ (C), 166.3 (C), 144.3 (C), 142.6 (C), 139.3 (C), $130.2(2 \mathrm{CH}), 130.1(\mathrm{C}), 128.4(2 \mathrm{CH}), 125.3(2 \mathrm{CH}), 119.8$ $(2 \mathrm{CH}), 76.3(\mathrm{C}), 74.8\left(\mathrm{CH}_{2}\right), 46.7(\mathrm{CH}), 44.7\left(\mathrm{CH}_{2}\right), 21.2$ $\left(\mathrm{CH}_{3}\right), 17.8\left(\mathrm{CH}_{2}\right)$. HPLC: Chiralpak IG eluted with 7:3 heptane/ethanol at $1 \mathrm{~mL} / \mathrm{min}$ at $25^{\circ} \mathrm{C}$, UV detection at $254 \mathrm{~nm}$, retention times: major enantiomer $11.23 \mathrm{~min}$, minor enantiomer $20.37 \mathrm{~min}$

\section{General Procedure for the Ring Rearrangement}

To a solution of 2-oxocyclobutane carboxamide 3 (ca $0.2 \mathrm{mmol}$ ) in anhydrous dichloromethane (ca. $2 \mathrm{~mL}$ ) was added the catalyst DHPB $(20 \mathrm{~mol} \%)$. The reaction was stirred at $28^{\circ} \mathrm{C}$ for the time indicated below with periodical monitoring by TLC analysis, concentrated under vacuum and directly purified by flash chromatography to give the glutarimide product 4 .

Compound 4 a: Following the general procedure, 3 a $(43 \mathrm{mg}$, $0.11 \mathrm{mmol}, \mathrm{dr}=20: 1$, er $>200: 1)$ reacted with DPHB $(4 \mathrm{mg}$, $0.021 \mathrm{mmol})$ for $8 \mathrm{~h}$ to provide $4 \mathbf{a}(39 \mathrm{mg}, 91 \%, \mathrm{dr}=2: 1$, er $=$ 199:1 and 111:1) as a white solid. $\boldsymbol{R}_{f}=0.30$ (petrol ether/ethyl acetate, 3:1). HRMS (ESI +$) \mathrm{m} / z$ calcd for $\mathrm{C}_{19} \mathrm{H}_{18} \mathrm{~N}_{3} \mathrm{O}_{6}{ }^{+}[\mathrm{M}+$ $\mathrm{H}]^{+}=384.1190$, found $=384.1190$. ${ }^{1} \mathbf{H} \quad \mathbf{N M R} \quad(400 \mathrm{MHz}$, $\mathrm{CDCl}_{3}$ ) major diastereomer: $\delta 8.32(\mathrm{~d}, J=9.0 \mathrm{~Hz}, 2 \mathrm{H}), 7.44$ $7.33(\mathrm{~m}, 3 \mathrm{H}), 7.29-7.21(\mathrm{~m}, 3 \mathrm{H}), 7.17(\mathrm{~d}, J=8.9 \mathrm{~Hz}, 1 \mathrm{H}), 5.22$ $(\mathrm{dd}, J=13.4,7.6 \mathrm{~Hz}, 1 \mathrm{H}), 5.02(\mathrm{dd}, J=13.3,7.6 \mathrm{~Hz}, 1 \mathrm{H}), 4.06$ (ddd, $J=7.6,7.6,3.5 \mathrm{~Hz}, 1 \mathrm{H}), 3.18$ (ddd, $J=12.0,5.1,3.6 \mathrm{~Hz}$, $1 \mathrm{H}), 2.84$ (ddd, $J=17.4,4.2,4.2 \mathrm{~Hz}, 1 \mathrm{H}), 2.79-2.63(\mathrm{~m}, 1 \mathrm{H})$, $2.22-2.10(\mathrm{~m}, 1 \mathrm{H}), 2.03(\mathrm{dd}, J=12.4,4.7 \mathrm{~Hz}, 1 \mathrm{H})$; minor diastereomer: $\delta 8.32(\mathrm{~d}, J=9.0 \mathrm{~Hz}, 2 \mathrm{H}), 7.44-7.33(\mathrm{~m}, 3 \mathrm{H})$, $7.29-7.21(\mathrm{~m}, 3 \mathrm{H}), 7.17$ (d, $J=8.9 \mathrm{~Hz}, 1 \mathrm{H}), 5.14$ (dd, $J=13.5$, $7.4 \mathrm{~Hz}, 1 \mathrm{H}$ ), 4.77 (dd, $J=13.5,7.3 \mathrm{~Hz}, 1 \mathrm{H}$ ), 4.17 (ddd, $J=7.5$, $7.5,7.5 \mathrm{~Hz}, 1 \mathrm{H}), 3.07$ (ddd, $J=8.9,8.9,4.9 \mathrm{~Hz}, 1 \mathrm{H}), 2.92$ (ddd, $J=17.9,6.8,4.8 \mathrm{~Hz}, 1 \mathrm{H}), 2.79-2.63(\mathrm{~m}, 1 \mathrm{H}), 1.97$ (dd, $J=$ $12.7, \quad 4.6 \mathrm{~Hz}, 1 \mathrm{H}), \quad 1.71-1.83 \quad(\mathrm{~m}, \quad 1 \mathrm{H}) .{ }^{13} \mathbf{C}\left\{{ }^{1} \mathbf{H}\right\} \quad$ NMR $(100 \mathrm{MHz}, d 8$-tetrahydrofuran) major diastereomer: $\delta 172.6$ (C), 170.7 (C), 147.5 (C), 142.0 (C), 137.7 (C), 130.1 (2CH), $128.7(2 \mathrm{CH}), 128.6(2 \mathrm{CH}), 127.7(\mathrm{CH}), 123.6(2 \mathrm{CH}), 76.4$ $\left(\mathrm{CH}_{2}\right), 45.9(\mathrm{CH}), 44.7(\mathrm{CH}), 32.2\left(\mathrm{CH}_{2}\right), 20.6\left(\mathrm{CH}_{2}\right)$; minor diastereomer: $\delta 172.7(\mathrm{C}), 170.7(\mathrm{C}), 147.5(\mathrm{C}), 142.0(\mathrm{C})$, $137.5(\mathrm{C}), 130.1(2 \mathrm{CH}), 128.6(2 \mathrm{CH}), 127.7(\mathrm{CH}), 123.6$ $(2 \mathrm{CH}), 77.6\left(\mathrm{CH}_{2}\right), 44.7(\mathrm{CH}), 43.1(\mathrm{CH}), 31.0\left(\mathrm{CH}_{2}\right), 20.1$ $\left(\mathrm{CH}_{2}\right)$. HPLC: Chiralpak IC eluted with 1:1 heptane/ethanol at $1 \mathrm{~mL} / \mathrm{min}$ at $25^{\circ} \mathrm{C}$, UV detection at 205 and $230 \mathrm{~nm}$, retention times: major diastereomer $8.10 \mathrm{~min}$ (major enantiomer) and $6.38 \mathrm{~min}$ (minor enantiomer), minor diastereomer $6.98 \mathrm{~min}$ (major enantiomer) and $9.13 \mathrm{~min}$ (minor enantiomer).

Compound 4 b: Following the general procedure, $3 \mathbf{b}(65 \mathrm{mg}$, $0.16 \mathrm{mmol}, \mathrm{dr}=26: 1, \mathrm{er}=49: 1)$ reacted with $\mathbf{D P H B}(6 \mathrm{mg}$, $0.032 \mathrm{mmol}$ ) for $48 \mathrm{~h}$ to provide $4 \mathrm{~b}(43 \mathrm{mg}, 66 \%, \mathrm{dr}=1.4: 1$, er $=39: 1$ and 21:1) as a white solid. $\boldsymbol{R}_{f}=0.35$ (petrol ether/ethyl acetate, 3:1). HRMS (ESI + ) $m / z$ calcd for $\mathrm{C}_{22} \mathrm{H}_{23} \mathrm{~N}_{2} \mathrm{O}_{6}{ }^{+}[\mathrm{M}+$ $\mathrm{H}^{+}=411.1551$, found $=411.1552 .{ }^{1} \mathbf{H} \quad \mathbf{N M R} \quad(400 \mathrm{MHz}$, $\mathrm{CDCl}_{3}$ ) major diastereomer: $\delta 8.14(\mathrm{~d}, J=8.9 \mathrm{~Hz}, 2 \mathrm{H}), 7.44$ $7.20(\mathrm{~m}, 5 \mathrm{H}), 7.05(\mathrm{~d}, J=8.9 \mathrm{~Hz}, 2 \mathrm{H}), 5.20(\mathrm{dd}, J=13.5$, $7.1 \mathrm{~Hz}, 1 \mathrm{H}), 5.05(\mathrm{dd}, J=13.5,8.1 \mathrm{~Hz}, 1 \mathrm{H}), 4.39$ (q, $J=7.1 \mathrm{~Hz}$, 2H), 4.02 (ddd, $J=7.6,7.6,3.6 \mathrm{~Hz}, 1 \mathrm{H}), 3.16$ (ddd, $J=12.0$, $5.1,3.7 \mathrm{~Hz}, 1 \mathrm{H}$ ), 2.78 (ddd, $J=12.5,8.4,4.3 \mathrm{~Hz}, 1 \mathrm{H}$ ), 2.74 $2.59(\mathrm{~m}, 1 \mathrm{H}), 2.15-1.67(\mathrm{~m}, 2 \mathrm{H}), 1.39(\mathrm{t}, J=7.1 \mathrm{~Hz}, 3 \mathrm{H})$; minor diastereomer: $\delta 8.14(\mathrm{~d}, J=8.9 \mathrm{~Hz}, 2 \mathrm{H}), 7.44-7.20(\mathrm{~m}$, $5 \mathrm{H}), 7.12(\mathrm{~d}, J=8.9 \mathrm{~Hz}, 2 \mathrm{H}), 5.16(\mathrm{dd}, J=13.3,6.9 \mathrm{~Hz}, 1 \mathrm{H})$, $4.78(\mathrm{dd}, J=13.3,8.0 \mathrm{~Hz}, 1 \mathrm{H}), 4.39(\mathrm{q}, J=7.1 \mathrm{~Hz}, 2 \mathrm{H}), 4.16$ (dd, $J=15.3,8.0 \mathrm{~Hz}, 1 \mathrm{H}), 3.03$ (ddd, $J=9.3,9.3,4.9 \mathrm{~Hz}, 1 \mathrm{H}$ ), 2.88 (ddd, $J=17.9,6.5,4.8 \mathrm{~Hz}, 1 \mathrm{H}), 2.74-2.59$ (m, 1H), 2.15$1.67(\mathrm{~m}, 2 \mathrm{H}), 1.39(\mathrm{t}, \quad J=7.1 \mathrm{~Hz}, 3 \mathrm{H}) .{ }^{13} \mathbf{C}\left\{{ }^{1} \mathbf{H}\right\} \quad \mathbf{N M R}$ $\left(100 \mathrm{MHz}, \mathrm{CDCl}_{3}\right)$ major diastereomer: $\delta 172.8(\mathrm{C}), 171.2(\mathrm{C})$, 165.7 (C), 139.0 (C), 136.0 (C), 130.9 (C), 130.6 (2CH), 129.2 $(2 \mathrm{CH}), 128.8(2 \mathrm{CH}), 128.7(\mathrm{CH}), 128.5(2 \mathrm{CH}), 78.1\left(\mathrm{CH}_{2}\right)$, $61.2\left(\mathrm{CH}_{2}\right), 45.4(\mathrm{CH}), 44.8\left(\mathrm{CH}_{2}\right), 32.3\left(\mathrm{CH}_{2}\right), 21.5,\left(\mathrm{CH}_{2}\right)$ $14.3\left(\mathrm{CH}_{3}\right)$; minor diastereomer: $\delta 172.9(\mathrm{C}), 171.2(\mathrm{C}), 165.7$ $(\mathrm{C}), 139.0(\mathrm{C}), 136.3(\mathrm{C}), 130.9(\mathrm{C}), 129.4(2 \mathrm{CH}), 128.6(2 \mathrm{CH})$, $128.7(\mathrm{CH}), 128.6(2 \mathrm{CH}), 128.2(2 \mathrm{CH}), 77.2\left(\mathrm{CH}_{2}\right), 61.2\left(\mathrm{CH}_{2}\right)$, $44.8(\mathrm{CH}), 43.7\left(\mathrm{CH}_{2}\right), 31.2\left(\mathrm{CH}_{2}\right), 20.5\left(\mathrm{CH}_{2}\right), 14.3\left(\mathrm{CH}_{3}\right)$. HPLC: Chiralpak IA eluted with 1:1 heptane/ethanol at $1 \mathrm{~mL} /$ min at $25^{\circ} \mathrm{C}$, UV detection at $220 \mathrm{~nm}$, retention times: major diastereomer $22.26 \mathrm{~min}$ (major enantiomer) and $35.51 \mathrm{~min}$ (minor enantiomer), minor diastereomer $41.26 \mathrm{~min}$ (major enantiomer) and $32.57 \mathrm{~min}$ (minor enantiomer).

Compound 4c: Following the general procedure, 3c $(50 \mathrm{mg}$, $0.14 \mathrm{mmol}, \mathrm{dr}=20: 1, \mathrm{er}=56: 1)$ reacted with DPHB $(5 \mathrm{mg}$, $0.026 \mathrm{mmol})$ for $72 \mathrm{~h}$ to provide $4 \mathrm{c}(24 \mathrm{mg}, 48 \%, \mathrm{dr}=1.6: 1$, er $=40: 1$ and 13:1) as a white solid. $\boldsymbol{R}_{f}=0.28$ (petrol ether/ethyl acetate, 3:1). HRMS (ESI + ) $m / z$ calcd for $\mathrm{C}_{20} \mathrm{H}_{21} \mathrm{~N}_{4} \mathrm{O}_{4}{ }^{+}[\mathrm{M}+$ $\left.\mathrm{NH}_{4}\right]^{+}=381.1557$, found $=381.1556$. ${ }^{1} \mathbf{H}$ NMR $(400 \mathrm{MHz}$, $\mathrm{CDCl}_{3}$ ) major diastereomer: $\delta 7.74(\mathrm{~d}, J=8.7 \mathrm{~Hz}, 2 \mathrm{H}), 7.42-$ $7.32(\mathrm{~m}, 3 \mathrm{H}), 7.26-7.20(\mathrm{~m}, 2 \mathrm{H}), 7.11(\mathrm{~d}, J=8.7 \mathrm{~Hz}, 2 \mathrm{H}), 5.20$ 
(dd, $J=13.4,7.5 \mathrm{~Hz}, 1 \mathrm{H}), 5.02(\mathrm{dd}, J=13.3,7.7 \mathrm{~Hz}, 1 \mathrm{H}), 4.05$ (ddd, $J=7.6,3.6,3.6 \mathrm{~Hz}, 1 \mathrm{H}), 3.16$ (ddd, $J=12.0,5.1,3.6 \mathrm{~Hz}$, $1 \mathrm{H}), 2.81$ (ddd, $J=17.4,4.3,4.3 \mathrm{~Hz}, 1 \mathrm{H}), 2.77-2.64(\mathrm{~m}, 1 \mathrm{H})$, 2.19-2.09 (m, 1H), 2.06-1.92 (m, 1H); minor diastereomer: $\delta 7.75(\mathrm{~d}, J=8.7 \mathrm{~Hz}, 2 \mathrm{H}), 7.44-7.32(\mathrm{~m}, 3 \mathrm{H}), 7.24-7.20(\mathrm{~m}$, 2H), $7.18(\mathrm{~d}, J=8.7 \mathrm{~Hz}, 2 \mathrm{H}), 5.14(\mathrm{dd}, J=13.5,7.3 \mathrm{~Hz}, 1 \mathrm{H})$, 4.76 (dd, $J=13.5,7.4 \mathrm{~Hz}, 1 \mathrm{H}), 4.16$ (ddd, $J=7.5,7.5,7.5 \mathrm{~Hz}$, $1 \mathrm{H}), 3.05$ (ddd, $J=8.9,8.9,4.9 \mathrm{~Hz}, 1 \mathrm{H}), 2.90$ (ddd, $J=17.9$, $6.8,4.8 \mathrm{~Hz}, 1 \mathrm{H}), 2.77-2.64(\mathrm{~m}, 1 \mathrm{H}), 2.06-1.92(\mathrm{~m}, 1 \mathrm{H}), 1.79$ $1.70(\mathrm{~m}, 1 \mathrm{H}) .{ }^{13} \mathbf{C}\left\{{ }^{1} \mathbf{H}\right\} \quad \mathbf{N M R}\left(100 \mathrm{MHz}, \mathrm{CDCl}_{3}\right)$ major diastereomer: $\delta 172.7(\mathrm{C}), 171.1(\mathrm{C}), 139.2$ (C), 136.1 (C), $133.2(2 \mathrm{CH}), 129.8(2 \mathrm{CH}), 129.4(2 \mathrm{CH}), 128.9(\mathrm{CH}), 128.8$ $(2 \mathrm{CH}), 118.1(\mathrm{C}), 113.0(\mathrm{C}), 77.3\left(\mathrm{CH}_{2}\right), 45.8(\mathrm{CH}), 45.5(\mathrm{CH})$ $32.4\left(\mathrm{CH}_{2}\right), 21.5\left(\mathrm{CH}_{2}\right)$; minor diastereomer: $\delta 172.9(\mathrm{C}), 171.2$ (C), 139.2 (C), 136.3 (C), $133.2(2 \mathrm{CH}), 129.8(2 \mathrm{CH}), 129.6$ $(2 \mathrm{CH}), 128.8(\mathrm{CH}), 128.3(2 \mathrm{CH}), 118.2(\mathrm{C}), 113.0(\mathrm{C}), 78.0$ $\left(\mathrm{CH}_{2}\right), 45.0(\mathrm{CH}), 43.6(\mathrm{CH}), 31.1\left(\mathrm{CH}_{2}\right), 20.5\left(\mathrm{CH}_{2}\right)$. HPLC: Chiralpak IC eluted with $7: 3$ heptane/ethanol at $1 \mathrm{~mL} / \mathrm{min}$ at $25^{\circ} \mathrm{C}$, UV detection at $220 \mathrm{~nm}$, retention times: major diastereomer $17.05 \mathrm{~min}$ (major enantiomer) and $11.83 \mathrm{~min}$ (minor enantiomer), minor diastereomer 13.49 min (major enantiomer) and $19.19 \mathrm{~min}$ (minor enantiomer).

Compound 4d: Following the general procedure, $3 \mathbf{d}(72 \mathrm{mg}$, $0.18 \mathrm{mmol}, \mathrm{dr}=22: 1, \mathrm{er}=55: 1)$ reacted with DPHB $(7 \mathrm{mg}$, $0.037 \mathrm{mmol})$ for $72 \mathrm{~h}$ to provide $4 \mathbf{d}(54 \mathrm{mg}, 75 \%, \mathrm{dr}=1.6: 1$, er $=43: 1$ and 21:1) as a white solid. $\boldsymbol{R}_{\boldsymbol{f}}=0.45$ (petrol ether/ethyl acetate, $3: 1)$. HRMS (ESI + ) $m / z$ calcd for $\mathrm{C}_{20} \mathrm{H}_{18} \mathrm{~F}_{3} \mathrm{~N}_{2} \mathrm{O}_{4}{ }^{+}[\mathrm{M}$ $+\mathrm{H}]^{+}=407.1213$, found $=407.1213$. ${ }^{1} \mathbf{H} \quad \mathbf{N M R}(400 \mathrm{MHz}$ $\left.\mathrm{CDCl}_{3}\right)$ major diastereomer: $\delta 7.75(\mathrm{~d}, J=8.7 \mathrm{~Hz}, 2 \mathrm{H}), 7.43$ 7.38 (m, 3H), 7.24-7.33 (m, 2H), 7.14 (d, $J=8.7 \mathrm{~Hz}, 2 \mathrm{H}), 5.23$ (dd, $J=13.4,7.3 \mathrm{~Hz}, 1 \mathrm{H}), 5.07(\mathrm{dd}, J=13.4,7.9 \mathrm{~Hz}, 1 \mathrm{H}), 4.05$ (ddd, $J=7.6,7.6,3.6 \mathrm{~Hz}, 1 \mathrm{H}$ ), 3.19 (ddd, $J=12.0,5.1,3.6 \mathrm{~Hz}$, $1 \mathrm{H}), 2.83$ (ddd, $J=17.4,4.3,4.3 \mathrm{~Hz}, 1 \mathrm{H}), 2.76-2.62(\mathrm{~m}, 1 \mathrm{H})$, 2.14 (ddd, $J=17.5,9.1,5.2 \mathrm{~Hz}, 1 \mathrm{H}), 2.06-1.93(\mathrm{~m}, 1 \mathrm{H})$; minor diastereomer: $\delta 7.75(\mathrm{~d}, J=8.7 \mathrm{~Hz}, 2 \mathrm{H}), 7.43-7.38(\mathrm{~m}, 3 \mathrm{H})$, $7.24-7.33(\mathrm{~m}, 2 \mathrm{H}), 7.21(\mathrm{~d}, J=8.7 \mathrm{~Hz}, 2 \mathrm{H}), 5.17(\mathrm{dd}, J=13.4$ $7.1 \mathrm{~Hz}, 1 \mathrm{H}), 4.79$ (dd, $J=13.4,7.7 \mathrm{~Hz}, 1 \mathrm{H}), 4.19$ (dd, $J=15.4$, $7.6 \mathrm{~Hz}, 1 \mathrm{H}), 3.08$ (ddd, $J=9.2,9.2,4.9 \mathrm{~Hz}, 1 \mathrm{H}), 2.93(\mathrm{ddd}, J=$ $17.9,6.7,4.8 \mathrm{~Hz}, 1 \mathrm{H}), 2.76-2.66(\mathrm{~m}, 1 \mathrm{H}), 2.06-1.93(\mathrm{~m}, 1 \mathrm{H})$, 1.77 (ddd, $J=14.2,9.6,4.7 \mathrm{~Hz}, 1 \mathrm{H}) .{ }^{13} \mathbf{C}\left\{{ }^{1} \mathbf{H}\right\}$ NMR $(100 \mathrm{MHz}$, $\mathrm{CDCl}_{3}$ ) major diastereomer: $\delta 172.9(\mathrm{C}), 171.2(\mathrm{C}), 138.3(\mathrm{C})$, 136.1 (C), 131.1 (q, $\left.J_{C-F}=32.6 \mathrm{~Hz}, \mathrm{C}\right), 129.4(2 \mathrm{CH}), 129.1$ $(2 \mathrm{CH}), 128.9(2 \mathrm{CH}), 128.8(\mathrm{CH}), 126.6\left(\mathrm{q}, J_{C-F}=3.7 \mathrm{~Hz}, 2 \mathrm{CH}\right)$, $123.8\left(\mathrm{q}, J_{C-F}=270 \mathrm{~Hz}, \mathrm{CF}_{3}\right), 77.4\left(\mathrm{CH}_{2}\right), 45.9(\mathrm{CH}), 45.5$ $(\mathrm{CH}), 32.4\left(\mathrm{CH}_{2}\right), 21.6\left(\mathrm{CH}_{2}\right)$; minor diastereomer: $\delta 173.0(\mathrm{C})$, 171.3 (C), 138.2 (C), 136.4 (C), 131.1 (q, $\left.J_{C-F}=32.6 \mathrm{~Hz}, \mathrm{C}\right)$, $129.5(2 \mathrm{CH}), 129.2(2 \mathrm{CH}), 128.7(\mathrm{CH}), 128.3(2 \mathrm{CH}), 126.5(\mathrm{q}$, $\left.J_{C-F}=3.7 \mathrm{~Hz}, 2 \mathrm{CH}\right), 123.7$ (q, $\left.J_{C-F}=270 \mathrm{~Hz}, \mathrm{CF}_{3}\right), 78.1\left(\mathrm{CH}_{2}\right)$, $\left.45.0(\mathrm{CH}), 43.7(\mathrm{CH}), 31.2\left(\mathrm{CH}_{2}\right), 20.6\left(\mathrm{CH}_{2}\right) .{ }^{19} \mathbf{F}{ }^{13} \mathbf{C}\right\} \mathbf{N M R}$ $\left(282 \mathrm{MHz}, \mathrm{CDCl}_{3}\right)$ major diastereomer: $\delta-62.7$; minor diastereomer: $\delta-62.7$. HPLC: Chiralpak IC eluted with 8:2 heptane/ ethanol at $1 \mathrm{~mL} / \mathrm{min}$ at $25^{\circ} \mathrm{C}$, UV detection at $235 \mathrm{~nm}$, retention times: major diastereomer $11.67 \mathrm{~min}$ (major enantiomer) and $8.25 \mathrm{~min}$ (minor enantiomer), minor diastereomer $9.46 \mathrm{~min}$ (major enantiomer) and $15.33 \mathrm{~min}$ (minor enantiomer).

Compound $4 \mathrm{e}$ : Following the general procedure, 3 e $(91 \mathrm{mg}$, $0.19 \mathrm{mmol}, \mathrm{dr}=25: 1, \mathrm{er}=58: 1)$ reacted with DPHB $(7 \mathrm{mg}$, $0.037 \mathrm{mmol})$ for $24 \mathrm{~h}$ to provide $4 \mathrm{e}(77 \mathrm{mg}, 85 \%, \mathrm{dr}=1.5: 1$, er $=44: 1$ and $38: 1$ ) as a colorless oil. $\boldsymbol{R}_{\boldsymbol{f}}=0.60$ (petrol ether/ ethyl acetate, 3:1). HRMS (ESI + ) $m / z$ calcd for $\mathrm{C}_{21} \mathrm{H}_{20}$
$\mathrm{F}_{6} \mathrm{~N}_{3} \mathrm{O}_{4}{ }^{+}\left[\mathrm{M}+\mathrm{NH}_{4}\right]^{+}=492.1353$, found $=492.1350 .{ }^{1} \mathbf{H} \mathbf{~ N M R}$ $\left(400 \mathrm{MHz}, \mathrm{CDCl}_{3}\right)$ major diastereomer: $\delta 7.92(\mathrm{~s}, 1 \mathrm{H}), 7.53-$ $7.35(\mathrm{~m}, 5 \mathrm{H}), 7.26-7.19(\mathrm{~m}, 2 \mathrm{H}), 5.21(\mathrm{dd}, J=13.4,7.5 \mathrm{~Hz}$, $1 \mathrm{H}), 5.03(\mathrm{dd}, J=13.4,7.8 \mathrm{~Hz}, 1 \mathrm{H}), 4.03$ (ddd, $J=7.6,7.6$, $3.5 \mathrm{~Hz}, 1 \mathrm{H}), 3.18(\mathrm{ddd}, J=11.9,5.2,3.5 \mathrm{~Hz}, 1 \mathrm{H}), 2.82(\mathrm{dd}, J=$ 17.4, 4.2, 4.2 Hz, 1H), 2.77-2.61 (m, 1H), 2.23-2.12 (m, 1H), 2.07-1.90 (m, 1H); minor diastereomer: $\delta 7.92(\mathrm{~s}, 1 \mathrm{H}), 7.53-$ $7.35(\mathrm{~m}, 5 \mathrm{H}), 7.26-7.19(\mathrm{~m}, 2 \mathrm{H}), 5.14(\mathrm{dd}, J=13.4,7.3 \mathrm{~Hz}$, $1 \mathrm{H}), 4.78$ (dd, $J=13.4,7.6 \mathrm{~Hz}, 1 \mathrm{H}), 4.19$ (ddd, $J=7.6,7.6$, $7.6 \mathrm{~Hz}, 1 \mathrm{H}), 3.06$ (ddd, $J=9.7,8.1,4.9 \mathrm{~Hz}, 1 \mathrm{H}), 2.91$ (ddd, $J=$ $17.9,6.2,4.7 \mathrm{~Hz}, 1 \mathrm{H}), 2.77-2.61(\mathrm{~m}, 1 \mathrm{H}), 2.07-1.90(\mathrm{~m}, 1 \mathrm{H})$, 1.77 (dddd, $J=14.4,10.1,10.14 .6 \mathrm{~Hz}, 1 \mathrm{H}) .{ }^{13} \mathbf{C}\left\{{ }^{1} \mathbf{H}\right\}$ NMR $\left(100 \mathrm{MHz}, \mathrm{CDCl}_{3}\right)$ major diastereomer: $\delta 172.8(\mathrm{C}), 171.0(\mathrm{C})$, $136.5(\mathrm{C}), 135.8(\mathrm{C}), 132.8\left(\mathrm{q}, J_{C-F}=34.3 \mathrm{~Hz}, 2 \mathrm{CF}_{3}\right), 129.5$ $(2 \mathrm{CH}), 129.3(2 \mathrm{CH}), 128.9(\mathrm{CH}), 128.8(2 \mathrm{CH}), 122.8\left(\mathrm{q}, J_{C-F}=\right.$ $\left.273.7 \mathrm{~Hz}, 2 \mathrm{CF}_{3}\right), 122.8\left(\mathrm{q}, J_{\mathrm{C}-F}=3.7 \mathrm{~Hz}, \mathrm{CH}\right), 77.2\left(\mathrm{CH}_{2}\right), 45.9$ $(\mathrm{CH}), 45.5(\mathrm{CH}), 32.2\left(\mathrm{CH}_{2}\right), 21.4\left(\mathrm{CH}_{2}\right)$; minor diastereomer: $\delta 172.9(\mathrm{C}), 171.1(\mathrm{C}), 136.5(\mathrm{C}), 136.1(\mathrm{C}), 132.8$ (q, $J_{C-F}=$ $\left.34.3 \mathrm{~Hz}, 2 \mathrm{CF}_{3}\right), 129.4(2 \mathrm{CH}), 129.3(2 \mathrm{CH}), 128.7(2 \mathrm{CH}), 128.7$ $(\mathrm{CH}), 132.7\left(\mathrm{q}, J_{C-F}=272.7 \mathrm{~Hz}, 2 \mathrm{CF}_{3}\right), 122.8\left(\mathrm{q}, J_{C-F}=3.7 \mathrm{~Hz}\right.$, $\mathrm{CH}), 78.0\left(\mathrm{CH}_{2}\right), 44.9(\mathrm{CH}), 43.7(\mathrm{CH}), 31.3\left(\mathrm{CH}_{2}\right), 20.4\left(\mathrm{CH}_{2}\right)$. ${ }^{19} \mathbf{F}\left\{{ }^{13} \mathbf{C}\right\}$ NMR $\left(282 \mathrm{MHz}, \mathrm{CDCl}_{3}\right)$ major diastereomer: $\delta$ -62.8; minor diastereomer: $\delta-62.9$. HPLC: Chiralpak IF eluted with 9:1 heptane/ethanol at $1 \mathrm{~mL} / \mathrm{min}$ at $25^{\circ} \mathrm{C}$, UV detection at $210 \mathrm{~nm}$, retention times: major diastereomer $13.26 \mathrm{~min}$ (major enantiomer) and $6.20 \mathrm{~min}$ (minor enantiomer), minor diastereomer $7.38 \mathrm{~min}$ (major enantiomer) and $8.19 \mathrm{~min}$ (minor enantiomer).

Compound 4 i: Following the general procedure, $3 \mathbf{i}(65 \mathrm{mg}$, $0.16 \mathrm{mmol}, \mathrm{dr}=26: 1$, er $=49: 1)$ reacted with DPHB $(6 \mathrm{mg}$, $0.032 \mathrm{mmol})$ for $48 \mathrm{~h}$ to provide $4 \mathrm{i}(43 \mathrm{mg}, 66 \%, \mathrm{dr}=1.7: 1$, er $=12: 1$ and 19:1) as a white solid. $\boldsymbol{R}_{f}=0.30$ (petrol ether/ethyl acetate, 2:1). HRMS (ESI +$) \mathrm{m} / z$ calcd for $\mathrm{C}_{20} \mathrm{H}_{20} \mathrm{~N}_{3} \mathrm{O}_{6}{ }^{+}[\mathrm{M}+$ $\mathrm{H}]^{+}=398.1347$, found $=398.1345$. ${ }^{1} \mathbf{H} \quad \mathbf{N M R} \quad(400 \mathrm{MHz}$, $\left.\mathrm{CDCl}_{3}\right)$ major diastereomer: $\delta 8.32(\mathrm{~d}, J=8.7 \mathrm{~Hz}, 2 \mathrm{H}), 7.25-$ $7.09(\mathrm{~m}, 6 \mathrm{H}), 5.20(\mathrm{dd}, J=13.3,7.6 \mathrm{~Hz}, 1 \mathrm{H}), 4.99$ (dd, $J=$ 13.3, $7.7 \mathrm{~Hz}, 1 \mathrm{H}$ ), 4.00 (ddd, $J=7.7,7.7,3.6 \mathrm{~Hz}, 1 \mathrm{H}$ ), 3.15 (ddd, $J=11.8,5.2,3.5 \mathrm{~Hz}, 1 \mathrm{H}), 2.82$ (ddd, $J=17.5,4.4,4.4 \mathrm{~Hz}$, $1 \mathrm{H}), 2.72-2.63(\mathrm{~m}, 1 \mathrm{H}), 2.36(\mathrm{~s}, 3 \mathrm{H}), 2.20-2.12(\mathrm{~m}, 1 \mathrm{H}), 2.08-$ $1.94(\mathrm{~m}, 1 \mathrm{H})$; minor diastereomer: $\delta 8.32(\mathrm{~d}, J=8.7 \mathrm{~Hz}, 2 \mathrm{H})$, $7.25-7.10(\mathrm{~m}, 6 \mathrm{H}), 5.11(\mathrm{dd}, J=13.4,7.4 \mathrm{~Hz}, 1 \mathrm{H}), 4.74(\mathrm{dd}$, $J=13.4,7.4 \mathrm{~Hz}, 1 \mathrm{H}), 4.13$ (ddd, $J=7.6,7.6,7.6 \mathrm{~Hz}, 1 \mathrm{H}), 3.04$ (ddd, $J=8.7,7.6,4.9 \mathrm{~Hz}, 1 \mathrm{H}), 2.91$ (ddd, $J=18.0,7.0,4.7 \mathrm{~Hz}$, 1H), 2.78-2.72 (m, 1H), $2.36(\mathrm{~s}, 3 \mathrm{H}), 2.08-1.94(\mathrm{~m}, 1 \mathrm{H}), 1.77$ (dddd, $J=14.0,9.3,9.3,4.8 \mathrm{~Hz}, 1 \mathrm{H}) .{ }^{13} \mathbf{C}\left\{{ }^{1} \mathbf{H}\right\}$ NMR $(100 \mathrm{MHz}$, $\mathrm{CDCl}_{3}$ ) major diastereomer: $\delta 173.4(\mathrm{C}), 171.4(\mathrm{C}), 148.2(\mathrm{C})$, $142.8(\mathrm{C}), 138.2(\mathrm{C}), 135.3(\mathrm{C}), 130.9(2 \mathrm{CH}), 130.0(2 \mathrm{CH})$, $129.4(2 \mathrm{CH}), 124.4(2 \mathrm{CH}), 77.4\left(\mathrm{CH}_{2}\right), 46.7(\mathrm{CH}), 45.3(\mathrm{CH})$, $32.9\left(\mathrm{CH}_{2}\right), 21.4\left(\mathrm{CH}_{2}\right), 20.9\left(\mathrm{CH}_{3}\right)$; minor diastereomer: $\delta 173.5$ (C), 171.5 (C), 148.2 (C), 142.8 (C), 138.1 (C), 135.1 (C), $130.9(2 \mathrm{CH}), 130.0(2 \mathrm{CH}), 129.2(2 \mathrm{CH}), 124.4(2 \mathrm{CH})$, $78.5\left(\mathrm{CH}_{2}\right), 45.5(\mathrm{CH}), 43.6(\mathrm{CH}), 32.6\left(\mathrm{CH}_{2}\right), 20.9\left(\mathrm{CH}_{2}\right), 20.8$ $\left(\mathrm{CH}_{3}\right)$. HPLC: Chiralpak ID eluted with 1:1 heptane/ethanol at $1 \mathrm{~mL} / \mathrm{min}$ at $25^{\circ} \mathrm{C}$, UV detection at $260 \mathrm{~nm}$, retention time: major diastereomer $17.06 \mathrm{~min}$ (major enantiomer) and $9.31 \mathrm{~min}$ (minor enantiomer), minor diastereomer $13.02 \mathrm{~min}$ (major enantiomer) and 11.14 min (minor enantiomer).

Compound $4 \mathbf{j}$ : Following the general procedure, $3 \mathbf{j}$ (50 mg, $0.12 \mathrm{mmol}, \mathrm{dr}=15: 1$, er $>200: 1)$ reacted with DPHB $(5 \mathrm{mg}$, $0.026 \mathrm{mmol})$ for $18 \mathrm{~h}$ to provide $4 \mathbf{j}(38 \mathrm{mg}, 76 \%, \mathrm{dr}=2.2: 1$, 
er $=40: 1$ and 37:1) as a white solid. $\boldsymbol{R}_{f}=0.28$ (petrol ether/ethyl acetate, 3:1). HRMS $(E S I+) m / z$ calcd for $\mathrm{C}_{20} \mathrm{H}_{20} \mathrm{~N}_{3} \mathrm{O}_{7}{ }^{+}[\mathrm{M}+$ $\mathrm{H}^{+}=414.1296$, found $=414.1300 .{ }^{1} \mathbf{H} \quad \mathbf{N M R} \quad(400 \mathrm{MHz}$, $\left.\mathrm{CDCl}_{3}\right)$ major diastereomer: $\delta 8.31(\mathrm{~d}, J=8.7 \mathrm{~Hz}, 2 \mathrm{H}), 7.17(\mathrm{~d}$, $J=8.7 \mathrm{~Hz}, 2 \mathrm{H}), 7.16(\mathrm{~d}, J=8.7 \mathrm{~Hz}, 2 \mathrm{H}), 6.89(\mathrm{~d}, J=8.7 \mathrm{~Hz}$ $2 \mathrm{H}), 5.18$ (dd, $J=13.3,7.5 \mathrm{~Hz}, 1 \mathrm{H}), 5.00$ (dd, $J=13.3,7.8 \mathrm{~Hz}$, 1H), 3.97 (ddd, $J=7.7,7.7,3.6 \mathrm{~Hz}, 1 \mathrm{H}), 3.82$ (s, 3H), 3.14 (ddd, $J=11.8,5.1,3.6 \mathrm{~Hz}, 1 \mathrm{H}), 2.82$ (ddd, $J=17.4,4.3,4.3 \mathrm{~Hz}$ 1H), 2.72 (dddd, $J=12.6,9.0,5.8,5.8 \mathrm{~Hz}, 1 \mathrm{H}), 2.22-2.08(\mathrm{~m}$, $1 \mathrm{H}), 2.07-1.93(\mathrm{~m}, 1 \mathrm{H})$; minor diastereomer: $\delta 8.31(\mathrm{~d}, J=$ $8.7 \mathrm{~Hz}, 2 \mathrm{H}), 7.24$ (d, $J=8.7 \mathrm{~Hz}, 2 \mathrm{H}), 7.14$ (d, $J=8.7 \mathrm{~Hz}, 2 \mathrm{H})$, $6.91(\mathrm{~d}, J=8.7 \mathrm{~Hz}, 2 \mathrm{H}), 5.09(\mathrm{dd}, J=13.3,7.4 \mathrm{~Hz}, 1 \mathrm{H}), 4.73$ (dd, $J=13.3,7.5 \mathrm{~Hz}, 1 \mathrm{H}), 4.13$ (ddd, $J=7.6,7.6,7.6 \mathrm{~Hz}, 1 \mathrm{H})$, $3.81(\mathrm{~s}, 3 \mathrm{H}), 3.02$ (ddd, $J=9.0,9.0,4.9 \mathrm{~Hz}, 1 \mathrm{H}), 2.91$ (ddd, $J=$ $17.9,6.8,4.8 \mathrm{~Hz}, 1 \mathrm{H}$ ), 2.72 (dddd, $J=12.6,9.0,5.8,5.8 \mathrm{~Hz}$, 1H), 2.07-1.93 (m, 1H), 1.77 (dddd, $J=14.1,9.5,9.0,4.8 \mathrm{~Hz}$, 1H). ${ }^{13} \mathbf{C}\left\{{ }^{1} \mathbf{H}\right\}$ NMR $\left(100 \mathrm{MHz}, \mathrm{CDCl}_{3}\right)$ major diastereomer: $\delta 172.8$ (C), 171.1 (C), 159.9 (C), 147.8 (C), 140.8 (C), 130.0 $(2 \mathrm{CH}), 129.9(\mathrm{C}), 127.7(2 \mathrm{CH}), 124.6(2 \mathrm{CH}), 114.7(2 \mathrm{CH})$, $77.7\left(\mathrm{CH}_{2}\right), 55.5\left(\mathrm{CH}_{3}\right), 45.5(\mathrm{CH}), 45.4(\mathrm{CH}), 32.4\left(\mathrm{CH}_{2}\right), 21.7$ $\left(\mathrm{CH}_{2}\right)$; minor diastereomer: $\delta 172.9(\mathrm{C}), 171.2(\mathrm{C}), 159.8(\mathrm{C})$, 147.8 (C), 140.8 (C), 130.0 (2 CH), $129.4(\mathrm{C}), 127.9(2 \mathrm{CH})$ $124.6(2 \mathrm{CH}), 114.9(2 \mathrm{CH}), 78.1\left(\mathrm{CH}_{2}\right), 55.5\left(\mathrm{CH}_{3}\right), 45.1(\mathrm{CH})$, $42.9(\mathrm{CH}), 31.2\left(\mathrm{CH}_{2}\right), 20.4\left(\mathrm{CH}_{2}\right)$. HPLC: Chiralpak IF eluted with $1: 1$ heptane/ethanol at $1 \mathrm{~mL} / \mathrm{min}$ at $25^{\circ} \mathrm{C}$, UV detection at 260 and $280 \mathrm{~nm}$, retention time: major diastereomer $31.31 \mathrm{~min}$ (major enantiomer) and $16.87 \mathrm{~min}$ (minor enantiomer), minor diastereomer $21.34 \mathrm{~min}$ (major enantiomer) and $25.29 \mathrm{~min}$ (minor enantiomer)

Compound $4 \mathrm{k}$ : Following the general procedure, $3 \mathbf{k}(54 \mathrm{mg}$ $0.12 \mathrm{mmol}$, dr $>20: 1$, er $>200: 1)$ reacted with DPHB $(5 \mathrm{mg}$, $0.026 \mathrm{mmol})$ for $15 \mathrm{~h}$ to provide $4 \mathbf{k}(37 \mathrm{mg}, 69 \%, \mathrm{dr}=1.8: 1$, er $=75: 1$ and $6.4: 1$ ) as a white solid. $\boldsymbol{R}_{\boldsymbol{f}}=0.30$ (petrol ether/ ethyl acetate, 3:1). HRMS (ESI +) $\mathrm{m} / \mathrm{z}$ calcd for $\mathrm{C}_{19} \mathrm{H}_{20} \mathrm{BrN}_{4} \mathrm{O}_{6}{ }^{+}\left[\mathrm{M}+\mathrm{NH}_{4}\right]^{+}=479.0561$, found $=479.0562 .{ }^{1} \mathbf{H}$ NMR $\left(400 \mathrm{MHz}, \mathrm{CDCl}_{3}\right)$ major diastereomer: $\delta 8.32(\mathrm{~d}, J=$ $8.7 \mathrm{~Hz}, 2 \mathrm{H}), 7.52$ (d, $J=8.7 \mathrm{~Hz}, 2 \mathrm{H}), 7.17$ (d, $J=8.8 \mathrm{~Hz}, 2 \mathrm{H})$, $7.15(\mathrm{~d}, J=8.8 \mathrm{~Hz}, 2 \mathrm{H}), 5.17(\mathrm{dd}, J=13.5,7.2 \mathrm{~Hz}, 1 \mathrm{H}), 5.04$ (dd, $J=13.4,8.0 \mathrm{~Hz}, 1 \mathrm{H}), 4.01$ (ddd, $J=7.6,7.6,3.6 \mathrm{~Hz}, 1 \mathrm{H}$ ), 3.16 (ddd, $J=12.6,4.9,3.7 \mathrm{~Hz}, 1 \mathrm{H}), 2.98-2.84(\mathrm{~m}, 1 \mathrm{H}), 2.82-$ $2.68(\mathrm{~m}, 1 \mathrm{H}), 2.18-2.11(\mathrm{~m}, 1 \mathrm{H}), 2.08-1.88(\mathrm{~m}, 1 \mathrm{H})$; minor diastereomer: $\delta 8.32(\mathrm{~d}, J=8.7 \mathrm{~Hz}, 2 \mathrm{H}), 7.53(\mathrm{~d}, J=8.7 \mathrm{~Hz}$ $2 \mathrm{H}), 7.24$ (d, $J=8.8 \mathrm{~Hz}, 2 \mathrm{H}), 7.13(\mathrm{~d}, J=8.8 \mathrm{~Hz}, 2 \mathrm{H}), 5.12$ (dd, $J=13.4,7.3 \mathrm{~Hz}, 1 \mathrm{H}), 4.75(\mathrm{dd}, J=13.4,7.6 \mathrm{~Hz}, 1 \mathrm{H}), 4.18$ (ddd, $J=7.5,7.5,7.5 \mathrm{~Hz}, 1 \mathrm{H}$ ), 3.03 (ddd, $J=10.0,7.8,4.8 \mathrm{~Hz}$ $1 \mathrm{H}), 2.98-2.84(\mathrm{~m}, 1 \mathrm{H}), 2.73(\mathrm{dd}, J=12.9,4.9 \mathrm{~Hz}, 1 \mathrm{H}), 2.07-$ $1.90(\mathrm{~m}, 1 \mathrm{H}), 1.76$ (dddd, $J=14.7,10.2,10.2,4.7 \mathrm{~Hz}, 1 \mathrm{H}) .{ }^{13} \mathbf{C}$ $\left\{{ }^{1} \mathbf{H}\right\}$ NMR (100 MHz, d8-tetrahydrofuran) major diastereomer: $\delta 173.3(\mathrm{C}), 171.4(\mathrm{C}), 148.3(\mathrm{C}), 142.7$ (C), $137.8(\mathrm{C}), 132.5$ $(2 \mathrm{CH}), 131.6(2 \mathrm{CH}), 130.9(2 \mathrm{CH}), 124.4(2 \mathrm{CH}), 122.4(\mathrm{C})$ $77.0\left(\mathrm{CH}_{2}\right), 44.6(\mathrm{CH}), 45.0(\mathrm{CH}), 33.0\left(\mathrm{CH}_{2}\right), 21.4\left(\mathrm{CH}_{2}\right)$ minor diastereomer: $\delta 173.3(\mathrm{C}), 171.4(\mathrm{C}), 148.3(\mathrm{C}), 142.7$ (C), $134.5(\mathrm{C}), 132.5(2 \mathrm{CH}), 131.5(2 \mathrm{CH}), 130.9(2 \mathrm{CH}), 124.4$ $(2 \mathrm{CH}), 122.4(\mathrm{C}), 78.0\left(\mathrm{CH}_{2}\right), 45.3(\mathrm{CH}), 43.3(\mathrm{CH}), 32.0$ $\left(\mathrm{CH}_{2}\right), 20.8\left(\mathrm{CH}_{2}\right)$. HPLC: Chiralpak IC eluted with $7: 3$ heptane/ethanol at $1 \mathrm{~mL} / \mathrm{min}$ at $25^{\circ} \mathrm{C}$, UV detection at $280 \mathrm{~nm}$, retention times: major diastereomer $12.64 \mathrm{~min}$ (major enantiomer) and $9.45 \mathrm{~min}$ (minor enantiomer), minor diastereomer $10.38 \mathrm{~min}$ (major enantiomer) and $15.81 \mathrm{~min}$ (minor enantiomer).
Compound 41: Following the general procedure, 31 (50 mg, $0.12 \mathrm{mmol}, \mathrm{dr}>20: 1$, er $=39: 1)$ reacted with DPHB (5 mg, $0.026 \mathrm{mmol})$ for $15 \mathrm{~h}$ to provide $41(22 \mathrm{mg}, 44 \%, \mathrm{dr}>20: 1$, er $=50: 1$ ) as a white solid. $\boldsymbol{R}_{f}=0.36$ (petrol ether/ethyl acetate, $1: 1) \cdot[\boldsymbol{\alpha}]_{\mathbf{D}}{ }^{25}=+21.7\left(c=0.5, \mathrm{CHCl}_{3}\right)$. HRMS $(\mathrm{ESI}+) \mathrm{m} / \mathrm{z}$ calcd for $\mathrm{C}_{19} \mathrm{H}_{20} \mathrm{~N}_{5} \mathrm{O}_{8}{ }^{+}\left[\mathrm{M}+\mathrm{NH}_{4}\right]^{+}=446.1306$, found $=446.1307$. ${ }^{1} \mathbf{H}$ NMR $\left(400 \mathrm{MHz}, \mathrm{CDCl}_{3}\right): \delta 8.33(\mathrm{~d}, J=8.8 \mathrm{~Hz}, 2 \mathrm{H}), 8.25$ (d, $J=8.8 \mathrm{~Hz}, 2 \mathrm{H}), 7.50(\mathrm{~d}, J=8.8 \mathrm{~Hz}, 2 \mathrm{H}), 7.19(\mathrm{~d}, J=$ $8.8 \mathrm{~Hz}, 2 \mathrm{H}), 5.17(\mathrm{dd}, J=10.8,3.2 \mathrm{~Hz}, 1 \mathrm{H}), 5.14$ (dd, $J=10.8$, $2.8 \mathrm{~Hz}, 1 \mathrm{H}$ ), 4.21 (ddd, $J=7.4,3.8,3.8 \mathrm{~Hz}, 1 \mathrm{H}$ ), 3.23 (ddd, $J=$ 8.6, 4.6, 4.6 Hz, 1H), 2.97 (ddd, $J=17.7,4.4,2.9 \mathrm{~Hz}, 1 \mathrm{H}), 2.80$ (ddd, $J=17.7,13.5,5.3 \mathrm{~Hz}, 1 \mathrm{H}$ ), 2.18 (dddd, $J=13.1,5.1,5.1$, $2.9 \mathrm{~Hz}, 1 \mathrm{H}), 1.94$ (dddd, $J=13.3,13.3,13.3,4.5 \mathrm{~Hz}, 1 \mathrm{H}) .{ }^{13} \mathrm{C}$ $\left\{{ }^{1} \mathbf{H}\right\}$ NMR $(100 \mathrm{MHz}, d 6$-acetone): $\delta 173.7(\mathrm{C}), 172.2(\mathrm{C})$, 148.5 (C), 148.4 (C), 146.7 (C), 143.2 (C), 131.4 (2CH), 131.1 $(2 \mathrm{CH}), 124.8(2 \mathrm{CH}), 124.6(2 \mathrm{CH}), 77.2\left(\mathrm{CH}_{2}\right), 46.9(\mathrm{CH}), 44.9$ $(\mathrm{CH}), 33.2\left(\mathrm{CH}_{2}\right), 21.1\left(\mathrm{CH}_{2}\right)$. HPLC: Chiralpak IC eluted with 2:2:1 heptane/ethanol/chloroforme at $1 \mathrm{~mL} / \mathrm{min}$ at $25^{\circ} \mathrm{C}$, UV detection at $254 \mathrm{~nm}$, retention time: major enantiomer $10.63 \mathrm{~min}$, minor enantiomer $8.03 \mathrm{~min}$.

Compound $4 \mathrm{~m}$ : Following the general procedure, $3 \mathrm{~m}(60 \mathrm{mg}$, $0.16 \mathrm{mmol}, \mathrm{dr}=28: 1, \mathrm{er}=100: 1)$ reacted with DPHB $(6 \mathrm{mg}$, $0.032 \mathrm{mmol}$ ) for $15 \mathrm{~h}$ to provide $4 \mathrm{~m}(41 \mathrm{mg}, 68 \%, \mathrm{dr}=2.4: 1$, er $=39: 1$ and 5.5:1) as a white solid. $\boldsymbol{R}_{f}=0.30$ (petrol ether/ ethyl acetate, 3:1). HRMS (ESI + ) $\mathrm{m} / z$ calcd for $\mathrm{C}_{17} \mathrm{H}_{19} \mathrm{~N}_{4} \mathrm{O}_{6} \mathrm{~S}^{+}$ $\left[\mathrm{M}+\mathrm{NH}_{4}\right]^{+}=407.1020, \quad$ found $=407.1019 . \quad{ }^{1} \mathbf{H} \quad$ NMR $\left(400 \mathrm{MHz}, \mathrm{CDCl}_{3}\right)$ major diastereomer: $\delta 8.33(\mathrm{~d}, J=8.7 \mathrm{~Hz}$, 2H), 7.33-7.30 (m, 1H), 7.24 (d, $J=8.7 \mathrm{~Hz}, 2 \mathrm{H}), 7.04-6.94(\mathrm{~m}$, $2 \mathrm{H}), 5.23(\mathrm{dd}, J=13.5,7.3 \mathrm{~Hz}, 1 \mathrm{H}), 4.99$ (dd, $J=13.5,7.4 \mathrm{~Hz}$, $1 \mathrm{H}$ ), 4.32 (ddd, $J=7.4,7.4,3.3 \mathrm{~Hz}, 1 \mathrm{H}), 3.20$ (ddd, $J=12.4$, $5.2,3.3 \mathrm{~Hz}, 1 \mathrm{H}), 3.01-2.85(\mathrm{~m}, 1 \mathrm{H}), 2.83-2.73(\mathrm{~m}, 1 \mathrm{H}), 2.22-$ $2.14(\mathrm{~m}, 1 \mathrm{H}), 2.08-2.00(\mathrm{~m}, 1 \mathrm{H})$; minor diastereomer: $\delta 8.32$ $(\mathrm{d}, J=8.7 \mathrm{~Hz}, 2 \mathrm{H}), 7.33-7.30(\mathrm{~m}, 1 \mathrm{H}), 7.22(\mathrm{~d}, J=8.7 \mathrm{~Hz}, 2 \mathrm{H})$, $7.04-6.94(\mathrm{~m}, 2 \mathrm{H}), 5.03(\mathrm{dd}, J=13.1,8.3 \mathrm{~Hz}, 1 \mathrm{H}), 4.83$ (dd, $J=10.2,6.8 \mathrm{~Hz}, 1 \mathrm{H}), 4.69(\mathrm{dd}, J=14.6,6.5 \mathrm{~Hz}, 1 \mathrm{H}), 3.08-3.02$ $(\mathrm{m}, 1 \mathrm{H}), 3.00-2.88(\mathrm{~m}, 1 \mathrm{H}), 2.83-2.73(\mathrm{~m}, 1 \mathrm{H}), 2.13-2.09(\mathrm{~m}$, 1H), 1.95-1.85 (m, 1H). ${ }^{13} \mathbf{C}\left\{{ }^{1} \mathbf{H}\right\}$ NMR $(100 \mathrm{MHz}, d 8$-tetrahydrofuran) major diastereomer: $\delta 173.4(\mathrm{C}), 171.4(\mathrm{C}), 148.3$ (C), $142.6(\mathrm{C}), 140.0(\mathrm{C}), 130.9(2 \mathrm{CH}), 128.2(\mathrm{CH}), 127.4$ $(\mathrm{CH}), 126.3(\mathrm{CH}), 124.4(2 \mathrm{CH}), 79.1\left(\mathrm{CH}_{2}\right), 45.8(\mathrm{CH}), 41.9$ $(\mathrm{CH}), 33.0\left(\mathrm{CH}_{2}\right), 22.2\left(\mathrm{CH}_{2}\right)$; minor diastereomer: $\delta 173.0(\mathrm{C})$, 171.4 (C), 148.3 (C), 142.8 (C), 139.8 (C), 130.9 (2CH), 127.4 $(\mathrm{CH}), 127.3(\mathrm{CH}), 126.0(\mathrm{CH}), 124.4(2 \mathrm{CH}), 78.1\left(\mathrm{CH}_{2}\right), 46.0$ $(\mathrm{CH}), 39.3(\mathrm{CH}), 32.3\left(\mathrm{CH}_{2}\right), 20.1\left(\mathrm{CH}_{2}\right)$. HPLC: Chiralpak IC eluted with $3: 2$ heptane/ethanol at $1 \mathrm{~mL} / \mathrm{min}$ at $25^{\circ} \mathrm{C}$, UV detection $254 \mathrm{~nm}$, retention time: major diastereomer $9.82 \mathrm{~min}$ (major enantiomer) and $7.46 \mathrm{~min}$ (minor enantiomer), minor diastereomer $8.88 \mathrm{~min}$ (major enantiomer) and $12.51 \mathrm{~min}$ (minor enantiomer).

Compound 4n: Following the general procedure, 3n (70 mg, $0.19 \mathrm{mmol}, \mathrm{dr}=15: 1, \mathrm{er}=48: 1)$ reacted with DPHB $(7 \mathrm{mg}$, $0.037 \mathrm{mmol})$ for $15 \mathrm{~h}$ to provide $4 \mathrm{n}(57 \mathrm{mg}, 81 \%, \mathrm{dr}=2: 1$, er $=$ 28:1 and 46:1) as a white solid. $\boldsymbol{R}_{\boldsymbol{f}}=0.28$ (petrol ether/ethyl acetate, $3: 1)$. HRMS $(E S I+) m / z$ calcd for $\mathrm{C}_{17} \mathrm{H}_{19} \mathrm{~N}_{4} \mathrm{O}_{7}{ }^{+}[\mathrm{M}+$ $\left.\mathrm{NH}_{4}\right]^{+}=391.1248$, found $=391.1248$. ${ }^{1} \mathbf{H}$ NMR $(400 \mathrm{MHz}$, $\mathrm{CDCl}_{3}$ ) major diastereomer: $\delta 8.31(\mathrm{~d}, J=8.8 \mathrm{~Hz}, 2 \mathrm{H}), 7.43(\mathrm{br}$ s, 1H), 7.37 (br s, 1H), 7.19 (d, $J=8.8 \mathrm{~Hz}, 2 \mathrm{H}), 6.30$ (br s, 1H), $5.12(\mathrm{dd}, J=13.3,7.5 \mathrm{~Hz}, 1 \mathrm{H}), 4.91(\mathrm{dd}, J=13.3,7.6 \mathrm{~Hz}, 1 \mathrm{H})$, 3.89 (ddd, $J=7.5,7.5,3.0 \mathrm{~Hz}, 1 \mathrm{H}$ ), 3.11 (ddd, $J=12.2,5.6$, $3.1 \mathrm{~Hz}, 1 \mathrm{H}), 3.00-2.90(\mathrm{~m}, 1 \mathrm{H}), 2.85-2.71(\mathrm{~m}, 1 \mathrm{H}), 2.14-1.98$ 
(m, 2H); minor diastereomer: $\delta 8.30(\mathrm{~d}, J=8.8 \mathrm{~Hz}, 2 \mathrm{H}), 7.45$ (br s, 1H), 7.35 (br s, 1H), 7.21 (d, $J=8.8 \mathrm{~Hz}, 2 \mathrm{H}), 6.28$ (br s, $1 \mathrm{H}), 4.89$ (dd, $J=12.9,8.4 \mathrm{~Hz}, 1 \mathrm{H}), 4.71(\mathrm{dd}, J=12.9,7.1 \mathrm{~Hz}$, $1 \mathrm{H}), 4.35$ (dd, $J=13.5,7.5 \mathrm{~Hz}, 1 \mathrm{H}), 2.99$ (dd, $J=10.4,4.8 \mathrm{~Hz}$, $1 \mathrm{H}), 2.97-2.87(\mathrm{~m}, 1 \mathrm{H}), 2.85-2.71(\mathrm{~m}, 1 \mathrm{H}), 2.14-1.98(\mathrm{~m}, 1 \mathrm{H})$, 1.92-1.81 (m, 1H). ${ }^{13} \mathbf{C}\left\{{ }^{1} \mathbf{H}\right\}$ NMR $(100 \mathrm{MHz}, d 8$-tetrahydrofuran) major diastereomer: $\delta 173.7(\mathrm{C}), 171.4(\mathrm{C}), 148.2(\mathrm{C})$, $144.3(\mathrm{CH}), 142.7(\mathrm{CH}), 142.2(\mathrm{CH}), 130.9(2 \mathrm{CH}), 124.4$ $(2 \mathrm{CH}), 122.0(\mathrm{CH}), 111.1(\mathrm{CH}), 78.3\left(\mathrm{CH}_{2}\right), 45.7(\mathrm{CH}), 37.6$ $(\mathrm{CH}), 33.0\left(\mathrm{CH}_{2}\right), 22.2\left(\mathrm{CH}_{2}\right)$; minor diastereomer: $\delta 173.3(\mathrm{C})$, $171.4(\mathrm{C}), 148.2(\mathrm{C}), 144.2(\mathrm{CH}), 142.7(\mathrm{CH}), 141.9(\mathrm{CH})$, $130.9(2 \mathrm{CH}), 124.4(2 \mathrm{CH}), 121.6(\mathrm{CH}), 110.7(\mathrm{CH}), 77.4$ $\left(\mathrm{CH}_{2}\right), 45.1(\mathrm{CH}), 35.2(\mathrm{CH}), 32.3\left(\mathrm{CH}_{2}\right), 19.8\left(\mathrm{CH}_{2}\right)$. HPLC: Chiralpak IC eluted with $4: 1$ heptane/ethanol at $1 \mathrm{~mL} / \mathrm{min}$ at $25^{\circ} \mathrm{C}$, UV detection at $254 \mathrm{~nm}$, retention times: major diastereomer $24.80 \mathrm{~min}$ (major enantiomer) and $17.08 \mathrm{~min}$ (minor enantiomer), minor diastereomer $18.98 \mathrm{~min}$ (major enantiomer) and $43.32 \mathrm{~min}$ (minor enantiomer).

Compound 4o: Following the general procedure, 3 o $(89 \mathrm{mg}$, $0.22 \mathrm{mmol}, \mathrm{dr}=28: 1, \mathrm{er}=24: 1)$ reacted with DPHB $(8 \mathrm{mg}$, $0.042 \mathrm{mmol})$ for $24 \mathrm{~h}$ to provide $4 \mathrm{o}(59 \mathrm{mg}, 66 \%, \mathrm{dr}=1.9: 1$, er $=19: 1$ and 15:1) as a white solid. $\boldsymbol{R}_{f}=0.43$ (petrol ether/ethyl acetate, 3:1). HRMS (ESI + ) $\mathrm{m} / z$ calcd for $\mathrm{C}_{21} \mathrm{H}_{25} \mathrm{~N}_{4} \mathrm{O}_{6}{ }^{+}[\mathrm{M}+$ $\left.\mathrm{NH}_{4}\right]^{+}=429.1769$, found $=429.1770$. ${ }^{1} \mathbf{H}$ NMR $(400 \mathrm{MHz}$, $\left.\mathrm{CDCl}_{3}\right)$ major diastereomer: $\delta 8.30(\mathrm{~d}, J=9.0 \mathrm{~Hz}, 2 \mathrm{H}), 7.35$ $7.14(\mathrm{~m}, 7 \mathrm{H}), 4.76(\mathrm{dd}, J=12.6,6.8 \mathrm{~Hz}, 1 \mathrm{H}), 4.57-4.48(\mathrm{~m}$, $1 \mathrm{H}), 3.03-2.61(\mathrm{~m}, 6 \mathrm{H}), 2.17-1.64(\mathrm{~m}, 4 \mathrm{H})$; minor diastereomer: $\delta 8.31(\mathrm{~d}, J=9.0 \mathrm{~Hz}, 2 \mathrm{H}), 7.35-7.14(\mathrm{~m}, 7 \mathrm{H}), 4.62-4.48(\mathrm{~m}$, 2H), 3.15-3.05 (m, 1H), 3.03-2.61 (m, 5H), 2.17-1.64 (m, 4H). ${ }^{13} \mathbf{C}\left\{{ }^{1} \mathbf{H}\right\}$ NMR (100 MHz, d8-tetrahydrofuran) major diastereomer: $\delta 173.6(\mathrm{C}), 171.6(\mathrm{C}), 148.2(\mathrm{C}), 142.8(\mathrm{C}), 142.2(\mathrm{C})$, $130.9(2 \mathrm{CH}), 129.0(2 \mathrm{CH}), 129.0(2 \mathrm{CH}), 126.7(\mathrm{CH}), 124.3$ $(2 \mathrm{CH}), 77.9\left(\mathrm{CH}_{2}\right), 44.3(\mathrm{CH}), 38.9(\mathrm{CH}), 34.2\left(\mathrm{CH}_{2}\right), 33.3$ $\left(\mathrm{CH}_{2}\right), 31.7\left(\mathrm{CH}_{2}\right), 20.6\left(\mathrm{CH}_{2}\right)$; minor diastereomer: $\delta 173.5$ (C), 171.6 (C), 148.2 (C), 142.9 (C), 142.2 (C), 130.9 (2CH), $128.9(2 \mathrm{CH}), 128.8(2 \mathrm{CH}), 126.6(\mathrm{CH}), 124.3(2 \mathrm{CH}), 77.0$ $\left(\mathrm{CH}_{2}\right), 44.7(\mathrm{CH}), 38.2(\mathrm{CH}), 34.2\left(\mathrm{CH}_{2}\right), 33.1\left(\mathrm{CH}_{2}\right), 32.2$ $\left(\mathrm{CH}_{2}\right), 19.2\left(\mathrm{CH}_{2}\right)$. HPLC: Chiralpak IE eluted with $1: 1$ heptane/ethanol at $1 \mathrm{~mL} / \mathrm{min}$ at $25^{\circ} \mathrm{C}$, UV detection at $260 \mathrm{~nm}$, retention times: major diastereomer 9.27 min (major enantiomer) and $13.88 \mathrm{~min}$ (minor enantiomer), minor diastereomer $11.56 \mathrm{~min}$ (major enantiomer) and $19.83 \mathrm{~min}$ (minor enantiomer).

\section{Computational Work}

See the Supporting Information.

\section{Acknowledgements}

Financial support from the Agence Nationale de la Recherche (ANR-07-BLAN-0269), Aix-Marseille Université, Centrale Marseille, and the Centre National de la Recherche Scientifique (CNRS) is gratefully acknowledged. Y.-L.W. and Y.R. thank the China Scholarship Council for PhD grants (no. 201508330296 and 201204490081, respectively). We thank Dr. Nicolas Vanthuyne and Ms. Marion Jean (Aix-Marseille Université and CNRS) for HPLC analyses.

\section{References}

[1] (R)-Sesbanine: a) R. G. Powell, C. R. Smith, Jr., D. Weisleder, D. A. Muthard, J. Clardy, J. Am. Chem. Soc. 1979, 101, 2784; b) K. Tomioka, K. Koga, Tetrahedron Lett. 1980, 21, 2321. (S)-Julocrotine: c) L. L. Silva, A. C. Joussef, J. Nat. Prod. 2011, 74, 1531. ( \pm )-Pomalidomide: marketed under the trade name Imnovid (Europe and Russia) or Pomalyst (USA) by Celgene. ( \pm )Lenalidomide: marketed under the trade name Revlimid (Europe) by Celgene. ( \pm )-Aminoglutethimide: marketed under the trade name Cytraden by Novartis. For novel promising analogs of pomalidomide and lenalidomide: d) J. D. Hansen, K. Condroski, M. Correa, G. Muller, H. Man, A. Ruchelman, W. Zhang, F. Vocanson, T. Crea, W. Liu, G. Lu, F. Baculi, L. LeBrun, A. Mahmoudi, G. Carmel, M. Hickman, C.-C. Lu, J. Med. Chem. 2018, 61, 492. For more complex glutarimide-containing natural products: e) T. Kagata, S. Saito, H. Shigemori, A. Ohsaki, H. Ishiyama, T. Kubota, J. Kobayashi, J. Nat. Prod. 2006, 69, 1517; f) G.-B. Xu, L.-M. Li, T. Yang, G.-L. Zhang, G.-Y. Li, Org. Lett. 2012, 14, 6052.

[2] Selected syntheses of glutarimides in the racemic series: a) H. Takaya, K. Yoshida, K. Isozaki, H. Terai, S.-I. Murahashi, Angew. Chem. Int. Ed. 2003, 42, 3302; b) H.W. Chen, R.-T. Hsu, M.-Y. Chang, N.-C. Chang, Org. Lett. 2006, 8, 3033; c) J. Zhang, M. Senthilkumar, S. C. Ghosh, S. H. Hong, Angew. Chem. Int. Ed. 2010, 49, 6391.

[3] a) D. A. Greenhalgh, N. S. Simpkins, Synlett 2002, 12, 2074; b) T. A. Moss, D. M. Barber, A. F. Kyle, D. J. Dixon, Chem. Eur. J. 2013, 19, 3071; c) N. B. Bennett, D. C. Duquette, J. Kim, W. B. Liu, A. N. Marziale, D. C. Behenna, S. C. Virgil, B. M. Stoltz Chem. Eur. J. 2013, 19, 4414 .

[4] a) M. d. M. Sanchez Duque, O. Baslé, N. Isambert, A. Gaudel-Siri, Y. Génisson, J.-C. Plaquevent, J. Rodriguez, T. Constantieux, Org. Lett. 2011, 13, 3296; b) S. Goudedranche, X. Bugaut, T. Constantieux, D. Bonne, J. Rodriguez, Chem. Eur. J. 2014, 20, 410; c) K. Zhang, M. Meazza, V. Dočekal, M. E. Light, J. Veselý, R. Rios, Eur. J. Org. Chem. 2017, 1749; d) S. Mondal, A. Ghosh, S. Mukherjee, A. T. Biju Org. Lett. 2018, 20, 4499. e) A. Moyano, R. Rios Chem. Rev. 2011, 111, 4703.

[5] a) G. Kang, M. Yamagami, S. Vellalath, D. Romo, Angew. Chem. Int. Ed. 2018, 57, 6527; b) Y. Zhou, Y.-L. Wei, J. Rodriguez, Y. Coquerel, Angew. Chem. Int. Ed. 2019, 58, 456

[6] a) M. Presset, Y. Coquerel, J. Rodriguez, J. Org. Chem. 2009, 74, 415. For a recent review on the Wolff rearrangement: b) Y. Coquerel, J. Rodriguez in Molecular Rearrangements in Organic Synthesis (Ed.: C. Rojas), Wiley, Hoboken, 2015, Chap. 3, p. 59.

[7] D. Mailhol, M. d. M. Sanchez Duque, W. Raimondi, D. Bonne, T. Constantieux, Y. Coquerel, J. Rodriguez, $A d v$. Synth. Catal. 2012, 354, 3523.

[8] M. Kobayashi, S. Okamoto, Tetrahedron Lett. 2006, 47, 4347. 
[9] a) T. Boddaert, Y. Coquerel, J. Rodriguez, Adv. Synth Catal. 2009, 351, 1744-1748 (corrigendum: Adv. Synth. Catal. 2009, 351, 2541). b) T. Boddaert, Y. Coquerel, J. Rodriguez, Chem. Eur. J. 2011, 17, 2266-2271.

[10] Enantiopure thalidomide and analogs undergo rapid racemization at physiological $\mathrm{pH}$, see: $\mathrm{S}$. K. Teo, W. A. Colburn, W. G. Tracewell, K. A. Kook, D. I. Stirling, M. S. Jaworsky, M. A. Scheffler, S. D. Thomas, O. L. Laskin Clin. Pharmacokinet. 2004, 43, 311.

[11] For related postulated acyl isothiouronium intermediates, see: D. G. Stark, L. C. Morrill, D. B. Cordes, A. M. Z.
Slawin, T. J. C. O'Riordan, A. D. Smith, Chem. Asian J. 2016, 11, 395.

[12] For the relative nucleophilicities of the catalysts depicted in Scheme 3, see: https://www.cup.lmu.de/oc/mayr/reaktionsdatenbank. Regrettably, the $\mathrm{pKa}$ and Lewis basicities of these catalyst in dichloromethane are essentially unknown.

[13] D. Bensa, T. Constantieux, J. Rodriguez, Synthesis 2004, 923. 

